\title{
Intertwining operator superalgebras and vertex tensor categories for superconformal algebras, I
}

\author{
Yi-Zhi Huang and Antun Milas
}

\begin{abstract}
We construct the intertwining operator superalgebras and vertex tensor categories for the $N=1$ superconformal minimal models and other related models.
\end{abstract}

\section{Introduction}

Superconformal symmetries play a fundamental role in string theory since perturbative string theory is described by two-dimensional quantum field theories with superconformal symmetries. On the other hand, superconformal algebras also arise in mathematical structures, for example, in the quantum field theory structure underlying the moonshine module for the Monster finite simple group (see [FLM1], [B]], [FLM2], [DGM], [DGH] and [H3]) and in the study of mirror symmetry for Calabi-Yau manifolds (see [Ge1], [Ge2], [D], [LVW], GP and many other works by physicists discussed in the survey Grif). A complete mathematical understanding of superconformal field theories is needed in order to solve the related mathematical problems.

Intertwining operator (super)algebras and vertex tensor categories are equivalent essentially to genus-zero weakly-holomorphic conformal field theories in the sense of Segal [51] [52] satisfying additional properties (see [H4] and [H6]). The general theory of intertwining operator algebras, including a construction of intertwining operator algebras from representations of suitable vertex operator algebras, was developed by the first author in [H4], [H6] and [H7]. A tensor product theory for modules for a vertex operator algebra, 
including a construction of vertex tensor categories from categories of modules for vertex operator algebras, was developed by Lepowsky and the first author in [HL1]-[HL7] and [H1]. Besides giving genus-zero weakly-holomorphic conformal field theories, the theory of intertwining operator algebras is also closely related to the so called "boundary conformal field theories" first developed by Cardy in [C1] and C2, which have many applications in condensed matter physics and have recently been applied successfully (see, for example, [RS] and [FS]) to the study of D-branes in string theory. We expect that the theory of intertwining operator algebras will provide a mathematical foundation to a number of "world sheet" constructions in conformal field theories, boundary conformal field theories and D-branes.

In the present series of papers, we shall construct intertwining operator superalgebras and vertex tensor categories associated to the superconformal minimal models and other related models.

In this paper (Part I), we apply previously obtained results on representations of $N=1$ superconformal algebras and associated vertex operator algebras to show that the general theory for the construction of intertwining operator (super)algebras and the tensor product theory for modules for a vertex operator (super)algebra are applicable in this case. Therefore we obtain an intertwining operator superalgebra structure on the direct sum of all inequivalent irreducible modules for a minimal $N=1$ superconformal vertex operator superalgebra. We also obtain a vertex tensor category structure and consequently a braided tensor category structure associated to these models. The main work in this paper is to prove that the conditions to use the theory of intertwining operator algebras and the tensor product theory are satisfied for these models. These results are also generalized easily to a much more general class of vertex operator superalgebras.

The present paper is organized as follows: In Section 1, we recall the notion of $N=1$ superconformal vertex operator superalgebra. In Section 2, we recall and prove some basic results on representations of minimal $N=1$ superconformal vertex operator superalgebras and of vertex operator superalgebras in a much more general class. Section 3 is devoted to the proof of the convergence and extension properties for products of intertwining operators for minimal $N=1$ superconformal vertex operator superalgebras and for vertex operator superalgebras in the general class. The main tool is null vectors and differential equations. Our main results on the intertwining operator superalgebra structures and vertex tensor category structures are given in Section 4. In the appendix (Section 5), for one particular exam- 
ple, we give explicitly the calculations of the fusion rules, null vectors and differential equations.

Acknowledgment: The research of Y.-Z. H. is supported in part by NSF grant DMS-9622961.

\section{$1 \quad N=1$ superconformal vertex operator su- peralgebras}

In this section we recall the notion of $N=1$ superconformal vertex operator algebra and basic properties of such an algebra. These algebras have been studied extensively by physicists, and are formulated precisely by Kac and Wang [KWan and by Barron [Ba1] Ba2].

Definition 1.1 An $N=1$ superconformal vertex operator superalgebra is a vertex operator superalgebra $(V, Y, \mathbf{1}, \omega)$ together with an odd element $\tau$ called the Neveu-Schwarz element satisfying the following axiom: Let

$$
Y(\tau, x)=\sum_{n \in \mathbb{Z}} G(n+1 / 2) x^{-n-2} .
$$

Then the following $N=1$ Neveu-Schwarz relations hold: For $m, n \in \mathbb{Z}$,

$$
\begin{aligned}
{[L(m), L(n)] } & =(m-n) L(m+n)+\frac{c}{12}\left(m^{3}-m\right) \delta_{m+n, 0}, \\
{[L(m), G(n+1 / 2)] } & =\left(\frac{m}{2}-(n+1 / 2)\right) G(m+n+1 / 2), \\
{[G(m+1 / 2), G(n-1 / 2)] } & =2 L(m+n)+\frac{c}{3}\left(m^{2}+m\right) \delta_{m+n, 0},
\end{aligned}
$$

where $L(m), m \in \mathbb{Z}$, are the Virasoro operators on $V$ and $c$ is the central charge of $V$.

Modules and intertwining operators for an $N=1$ superconformal vertex operator superalgebra are modules and intertwining operators for the underlying vertex operator superalgebra.

The $N=1$ superconformal vertex operator superalgebra defined above is denoted by $(V, Y, \mathbf{1}, \tau)$ (without $\omega$ since $\omega=L(-2) \mathbf{1}=\frac{1}{2} G(-1 / 2) \tau$ ) or simply $V$. Note that a module $W$ for a vertex operator superalgebra (in particular the algebra itself) has a $\mathbb{Z}_{2}$-grading called sign in addition to the 
$\mathbb{C}$-grading by weights. We shall always use $W^{0}$ and $W^{1}$ to denote the even and odd subspaces of $W$. If $W$ is irreducible, there exists $h \in \mathbb{C}$ such that $W=W^{0} \oplus W^{1}$ where $W^{0}=\coprod_{n \in h+\mathbb{Z}} W_{(n)}$ and $W^{1}=\coprod_{n \in h+\mathbb{Z}+1 / 2} W_{(n)}$ are the even and odd parts of $W$, respectively. We shall always use the notation $|\cdot|$ to denote the map from the union of the even and odd subspaces of a vertex operator superalgebra or of a module for such an algebra to $\mathbb{Z}_{2}$ by taking the signs of elements in the union.

The notion of $N=1$ superconformal vertex operator superalgebra above was reformulated using odd formal variables by Barron. For a complete and detailed discussion, see [Ba1] and [Ba2]. Here we only give the parts we need in later sections.

For $l$ symbols $\varphi_{1}, \ldots, \varphi_{l}$, consider the exterior algebra of the vector space over $\mathbb{C}$ spanned by these symbols. We denote this exterior algebra by $\mathbb{C}\left[\varphi_{1}, \ldots, \varphi_{l}\right]$. For any vector space $E$, we use $E\left[\varphi_{1}, \ldots, \varphi_{l}\right]$ to denote the tensor product of $E$ and $\mathbb{C}\left[\varphi_{1}, \ldots, \varphi_{l}\right]$. In particular, if $E$ is the polynomial algebra $\mathbb{C}\left[x_{1}, \ldots, x_{k}\right]$ generated by formal variables $x_{1}, \ldots, x_{k}$ or the space $\mathbb{C}\left[\left[x_{1}, \ldots, x_{k}\right]\right]$ of formal Laurent series generated by these formal variables, we have the vector space $\mathbb{C}\left[x_{1}, \ldots, x_{k}\right]\left[\varphi_{1}, \ldots, \varphi_{l}\right]$ or $\mathbb{C}\left[\left[x_{1}, \ldots, x_{k}\right]\right]\left[\varphi_{1}, \ldots, \varphi_{l}\right]$. In this case, we call $x_{1}, \ldots, x_{k}$ and $\varphi_{1}, \ldots, \varphi_{l}$ even and odd formal variables, respectively. Note that any element of $\mathbb{C}\left[x_{1}, \ldots, x_{k}\right]\left[\varphi_{1}, \ldots, \varphi_{l}\right]$ is a linear combination of monomials in $x_{1}, \ldots, x_{k}$ and $\varphi_{1}, \ldots, \varphi_{l}$. For each monomial, the total order in $x_{1}, \ldots, x_{k}$ and $\varphi_{1}, \ldots, \varphi_{l}$ and the order in $\varphi_{1}, \ldots, \varphi_{l}$ modulo 2 give a $\mathbb{Z}$-grading called degree and a $\mathbb{Z}_{2}$-grading called sign, respectively, to $\mathbb{C}\left[x_{1}, \ldots, x_{k}\right]\left[\varphi_{1}, \ldots, \varphi_{l}\right]$. With the $\mathbb{Z}_{2}$-grading, $\mathbb{C}\left[x_{1}, \ldots, x_{k}\right]\left[\varphi_{1}, \ldots, \varphi_{l}\right]$ is an associative superalgebra. Similarly, $\mathbb{C}\left[\left[x_{1}, \ldots, x_{k}\right]\right]\left[\varphi_{1}, \ldots, \varphi_{l}\right]$ is also a superalgebra.

For any vector space $E$, consider the vector space

$$
\begin{gathered}
E\left[x_{1}, \ldots, x_{k}\right]\left[\varphi_{1}, \ldots, \varphi_{l}\right], \\
E\left[x_{1}, x_{1}^{-1}, \ldots, x_{k}, x_{k}^{-1}\right]\left[\varphi_{1}, \ldots, \varphi_{l}\right], \\
E\left[\left[x_{1}, \ldots, x_{k}\right]\right]\left[\varphi_{1}, \ldots, \varphi_{l}\right], \\
E\left[\left[x_{1}, x_{1}^{-1}, \ldots, x_{k}, x_{k}^{-1}\right]\right]\left[\varphi_{1}, \ldots, \varphi_{l}\right], \\
E\left\{x_{1}, \ldots, x_{k}\right\}\left[\varphi_{1}, \ldots, \varphi_{l}\right]
\end{gathered}
$$

and

$$
E\left(\left(x_{1}, \ldots, x_{k}\right)\right)\left[\varphi_{1}, \ldots, \varphi_{l}\right] .
$$

If $E$ is a $\mathbb{Z}_{2}$-graded vector space, then there are natural structures of modules over the ring $\mathbb{C}\left[x_{1}, \ldots, x_{k}\right]\left[\varphi_{1}, \ldots, \varphi_{l}\right]$ on these spaces. 
Let $(V, Y, \mathbf{1}, \tau)$ be an $N=1$ superconformal vertex operator superalgebra. We define the vertex operator map with odd variable

$$
\begin{aligned}
Y: V \otimes V & \rightarrow V((x))[\varphi] \\
u \otimes v & \mapsto Y(u,(x, \varphi)) v
\end{aligned}
$$

by

$$
Y(u,(x, \varphi)) v=Y(u, x) v+\varphi Y(G(-1 / 2) u, x) v
$$

for $u, v \in V$. (We use the same notation $Y$ to denote the vertex operator map and the vertex operator map with odd variable.) Then we have:

Proposition 1.2 The vertex operator map with odd variable satisfies the following properties:

1. The vacuum property:

$$
Y(\mathbf{1},(x, \varphi))=1
$$

where 1 on the right-hand side is the identity map on $V$.

2. The creation property: For any $v \in V$,

$$
\begin{gathered}
Y(v,(x, \varphi)) \mathbf{1} \in V[[x]][\varphi], \\
\lim _{(x, \varphi) \mapsto(0,0)} Y(v,(x, \varphi)) \mathbf{1}=v .
\end{gathered}
$$

3. The Jacobi identity: In (End $\left.V)\left[\left[x_{0}, x_{0}^{-1}, x_{1}, x_{1}^{-1}, x_{2}, x_{2}^{-1}\right]\right]\left[\varphi_{1}, \varphi_{2}\right]\right]$, we have

$$
\begin{aligned}
x_{0}^{-1} \delta & \left(\frac{x_{1}-x_{2}-\varphi_{1} \varphi_{2}}{x_{0}}\right) Y\left(u,\left(x_{1}, \varphi_{1}\right)\right) Y\left(v,\left(x_{2}, \varphi_{2}\right)\right) \\
& -(-1)^{|u||v|} x_{0}^{-1} \delta\left(\frac{x_{2}-x_{1}+\varphi_{1} \varphi_{2}}{-x_{0}}\right) Y\left(v,\left(x_{2}, \varphi_{2}\right)\right) Y\left(u,\left(x_{1}, \varphi_{1}\right)\right) \\
= & x_{2}^{-1} \delta\left(\frac{x_{1}-x_{0}-\varphi_{1} \varphi_{2}}{x_{2}}\right) Y\left(Y\left(u,\left(x_{0}, \varphi_{1}-\varphi_{2}\right)\right) v,\left(x_{2}, \varphi_{2}\right)\right)
\end{aligned}
$$

for $u, v \in V$ which are either even or odd.

4. The $G(-1 / 2)$-derivative property: For any $v \in V$,

$$
Y(G(-1 / 2) v,(x, \varphi))=\left(\frac{\partial}{\partial \varphi}+\varphi \frac{\partial}{\partial x}\right) Y(v,(x, \varphi)),
$$


5. The $L(-1)$-derivative property: For any $v \in V$,

$$
Y(L(-1) v,(x, \varphi))=\frac{\partial}{\partial x} Y(v,(x, \varphi)) .
$$

6. The skew-symmetry: For any $u, v \in V$ which are either even or odd,

$$
Y(u,(x, \varphi)) v=(-1)^{|u||v|} e^{x L(-1)+\varphi G(-1 / 2)} Y(v,(-x,-\varphi)) u .
$$

The proof of this result is straightforward and can be found in [Ba2].

We can also reformulate the data and axioms for modules and intertwining operators for an $N=1$ superconformal vertex operator superalgebra using odd variables. Since the goal of the present paper is to construct an algebra from intertwining operators for certain $N=1$ superconformal vertex operator superalgebras, we give the details of the corresponding reformulation of the data and axioms for intertwining operators using odd variables.

Let $W_{1}, W_{2}$ and $W_{3}$ be modules for an $N=1$ superconformal vertex operator superalgebra $V$ and $\mathcal{Y}$ an intertwining operator of type $\left(\begin{array}{c}W_{3} \\ W_{1} W_{2}\end{array}\right)$. We define the corresponding intertwining operator map with odd variable

$$
\begin{aligned}
\mathcal{Y}: W_{1} \otimes W_{2} & \rightarrow W_{3}\{x\}[\varphi] \\
w_{(1)} \otimes w_{(2)} & \mapsto \mathcal{Y}\left(w_{(1)},(x, \varphi)\right) w_{(2)}
\end{aligned}
$$

by

$$
\mathcal{Y}\left(w_{(1)},(x, \varphi)\right) w_{(2)}=\mathcal{Y}\left(w_{(1)}, x\right) w_{(2)}+\varphi \mathcal{Y}\left(G(-1 / 2) w_{(1)}, x\right) w_{(2)}
$$

for $u, v \in V$. Then we have:

Proposition 1.3 The intertwining operator map with odd variable satisfies the following properties:

1. The Jacobi identity: In $\operatorname{Hom}\left(W_{1} \otimes W_{2}, W_{3}\right)\left\{x_{0}, x_{1}, x_{2}\right\}\left[\varphi_{1}, \varphi_{2}\right]$, we have

$$
\begin{aligned}
x_{0}^{-1} \delta & \left(\frac{x_{1}-x_{2}-\varphi_{1} \varphi_{2}}{x_{0}}\right) Y\left(u,\left(x_{1}, \varphi_{1}\right)\right) \mathcal{Y}\left(w_{(1)},\left(x_{2}, \varphi_{2}\right)\right) \\
- & (-1)^{|u|\left|w_{(1)}\right|} x_{0}^{-1} \delta\left(\frac{x_{2}-x_{1}+\varphi_{1} \varphi_{2}}{-x_{0}}\right) \mathcal{Y}\left(w_{(1)},\left(x_{2}, \varphi_{2}\right)\right) Y\left(u,\left(x_{1}, \varphi_{1}\right)\right) \\
& =x_{2}^{-1} \delta\left(\frac{x_{1}-x_{0}-\varphi_{1} \varphi_{2}}{x_{2}}\right) \mathcal{Y}\left(Y\left(u,\left(x_{0}, \varphi_{1}-\varphi_{2}\right)\right) w_{(1)},\left(x_{2}, \varphi_{2}\right)\right)(1.3)
\end{aligned}
$$

for $u \in V$ and $w_{(1)} \in W_{1}$ which are either even or odd. 
2. The $G(-1 / 2)$-derivative property: For any $v \in V$,

$$
\mathcal{Y}\left(G(-1 / 2) w_{(1)},(x, \varphi)\right)=\left(\frac{\partial}{\partial \varphi}+\varphi \frac{\partial}{\partial x}\right) \mathcal{Y}\left(w_{(1)},(x, \varphi)\right),
$$

3. The $L(-1)$-derivative property: For any $v \in V$,

$$
\mathcal{Y}\left(L(-1) w_{(1)},(x, \varphi)\right)=\frac{\partial}{\partial x} \mathcal{Y}\left(w_{(1)},(x, \varphi)\right) .
$$

4. The skew-symmetry: There is a linear isomorphism

$$
\Omega: \mathcal{V}_{W_{1} W_{2}}^{W_{3}} \rightarrow \mathcal{V}_{W_{2} W_{1}}^{W_{3}}
$$

such that

$$
\begin{aligned}
& \Omega(\mathcal{Y})\left(w_{(1)},(x, \varphi)\right) w_{(2)} \\
& \left.\quad=(-1)^{\left|w_{(1)}\right|\left|w_{(2)}\right|} e^{x L(-1)+\varphi G(-1 / 2)} \mathcal{Y}\left(w_{(2)},\left(e^{-\pi i} x,-\varphi\right)\right)\right) w_{(1)}
\end{aligned}
$$

for $w_{(1)} \in W_{1}$ and $w_{(2)} \in W_{2}$ which are either even or odd.

The proof of this result is similar to the proof of Proposition 1.2 and is omitted.

\section{Minimal $N=1$ superconformal vertex op- erator superalgebras}

In this section, we recall the constructions and results on minimal $N=$ 1 superconformal vertex operator superalgebras and their representations. Some new results needed in later sections are also proved. We then introduce in this section a class of vertex operator superalgebras and generalize most of the results for minimal $N=1$ superconformal vertex operator superalgebras to algebras in this class. A large part of the material in this section is from KWan and $\mathrm{Ad}$.

The $N=1$ Neveu-Schwarz Lie superalgebra is the Lie superalgebra

$$
\mathfrak{n s}^{(1)}=\oplus_{n \in \mathbb{Z}} \mathbb{C} L_{n} \oplus \oplus_{n \in \mathbb{Z}} G_{n+1 / 2} \oplus \mathbb{C} C
$$


satisfying the following $N=1$ Neveu-Schwarz relations:

$$
\begin{aligned}
{\left[L_{m}, L_{n}\right] } & =(m-n) L_{m+n}+\frac{C}{12}\left(m^{3}-m\right) \delta_{m+n, 0}, \\
{\left[L_{m}, G_{n+1 / 2}\right] } & =\left(\frac{m}{2}-\left(n+\frac{1}{2}\right)\right) G_{m+n+1 / 2}, \\
{\left[G_{m+1 / 2}, G_{n-1 / 2}\right] } & =2 L_{m+n}+\frac{C}{3}\left(m^{2}+m\right) \delta_{m+n, 0}, \\
{\left[C, L_{m}\right] } & =0 \\
{\left[C, G_{m+1 / 2}\right] } & =0
\end{aligned}
$$

for $m, n \in \mathbb{Z}$. For simplicity, we shall simply denote the $N=1$ NeveuSchwarz Lie superalgebra by $\mathfrak{n s}$ in this paper.

Note that the elements $L_{0}, L_{1}, L_{-1}, G_{1 / 2}, G_{-1 / 2}$ of $\mathfrak{n s}$ span a subalgebra. It is known that this subalgebra is isomorphic to $\mathfrak{o s p}(2,1)$.

We now construct representations of the $N=1$ Neveu-Schwarz Lie superalgebra. Consider the two subalgebras

$$
\begin{aligned}
& \mathfrak{n s}^{+}=\oplus_{n>0} \mathbb{C} L_{n} \oplus \oplus_{n \geq 0} G_{n+1 / 2} \\
& \mathfrak{n s}^{-}=\oplus_{n<0} \mathbb{C} L_{n} \oplus \oplus_{n<0} G_{n+1 / 2}
\end{aligned}
$$

of $\mathfrak{n s}$. Let $U(\cdot)$ be the functor from the category of Lie superalgebras to the category of associative algebras obtained by taking the universal enveloping algebras of Lie superalgebras. For any representation of $\mathfrak{n s}$, we shall use $L(m)$ and $G(m+1 / 2), m \in \mathbb{Z}$, to denote the representation images of $L_{m}$ and $G_{m+1 / 2}$. For any $c, h \in \mathbb{C}$, the Verma module $M_{\mathfrak{n} \mathfrak{s}}(c, h)$ for $\mathfrak{n s}$ is a free $U\left(\mathfrak{n s}^{-}\right)$-module generated by $\mathbf{1}_{c, h}$ such that

$$
\begin{aligned}
\mathfrak{n s}^{+} \mathbf{1}_{c, h} & =0 \\
L(0) \mathbf{1}_{c, h} & =h \mathbf{1}_{c, h}, \\
C \mathbf{1}_{c, h} & =c \mathbf{1}_{c, h} .
\end{aligned}
$$

There exists a unique maximal proper submodule $J_{\mathfrak{n s}}(c, h)$ of $M_{\mathfrak{n s}}(c, h)$. It is easy to see that when $c \neq 0, \mathbf{1}_{c, 0}, G(-3 / 2) \mathbf{1}_{c, 0}$ and $L(-2) \mathbf{1}_{c, 0}$ are not in $J_{\mathfrak{n} \mathfrak{s}}(c, 0)$. Let

$$
L_{\mathfrak{n} \mathfrak{s}}(c, h)=M_{\mathfrak{n} \mathfrak{s}}(c, h) / J_{\mathfrak{n s}}(c, h)
$$

and

$$
V_{\mathfrak{n s}}(c, 0)=M_{\mathfrak{n s}}(c, 0) /\left\langle G(-1 / 2) \mathbf{1}_{c, 0}\right\rangle
$$


where $\left\langle G(-1 / 2) \mathbf{1}_{c, 0}\right\rangle$ is the submodule of $M_{\mathfrak{n s}}(c, 0)$ generated by $G(-1 / 2) \mathbf{1}_{c, 0}$. Then $L_{\mathfrak{n} \mathfrak{s}}(c, 0)$ and $V_{\mathfrak{n s}}(c, 0)$ have the structures of vertex operator superalgebras with the vacuum $\mathbf{1}_{c, 0}$, the Neveu-Schwarz element $G(-3 / 2) \mathbf{1}_{c, 0}$ and the Virasoro element $L(-2) \mathbf{1}_{c, 0}$ (see KWan).

The following result was conjectured by Kac and Wang in KWan and proved by Adamović in [Ad] using the relationship between representations of the $N=1$ Neveu-Schwarz Lie superalgebra and representations of the affine Lie algebra $A_{1}^{(1)}$ on the rational level obtained in [GKO], [KWak1], [KWak2], AM and As:

Theorem 2.1 The vertex operator superalgebra $L_{\mathfrak{n} \mathfrak{s}}(c, 0)$ has finitely many irreducible modules and every module for $L_{\mathfrak{n s}}(c, 0)$ is completely reducible if and only if

$$
c=c_{p, q}=\frac{3}{2}\left(1-2 \frac{(p-q)^{2}}{p q}\right)
$$

where $p, q$ are integers larger than 1 such that $p-q \in 2 \mathbb{Z}$ and $(p-q) / 2$ and $q$ are relatively prime to each other. A set of representatives of the equivalence classes of irreducible modules for $L_{\mathfrak{n} \mathfrak{s}}\left(c_{p, q}, 0\right)$ is

$$
\left\{L_{\mathfrak{n S}}\left(c_{p, q}, h_{p, q}^{m, n}\right)\right\}_{0<m<p, 0<n<q, m, n \in \mathbb{Z}, m-n \in 2 \mathbb{Z}}
$$

where for any $m, n \in \mathbb{Z}$ satisfying $0<m<p, 0<n<q$ and $m-n \in 2 \mathbb{Z}$,

$$
h_{p, q}^{m, n}=\frac{(n p-m q)^{2}-(p-q)^{2}}{8 p q} .
$$

For any pair $p, q$ of integers larger than 1 such that $p-q \in 2 \mathbb{Z}$ and $(p-q) / 2$ and $q$ are relatively prime to each other, we call the vertex operator algebra $L_{\mathfrak{n} \mathfrak{s}}\left(c_{p, q}, 0\right)$ a minimal $N=1$ superconformal vertex operator superalgebra.

Proposition 2.2 Let $m_{i}, n_{i} \in \mathbb{Z}, i=1,2,3$, satisfying $0<m_{i}<p, 0<n_{i}<$ $q$ and $m_{i}-n_{i} \in 2 \mathbb{Z}$ and $\mathcal{Y}$ an intertwining operator of type

$$
\left(\begin{array}{c}
L_{\mathfrak{n} \mathfrak{s}}\left(c_{p, q}, h_{p, q}^{m_{3}, n_{3}}\right) \\
L_{\mathfrak{n s}}\left(c_{p, q}, h_{p, q}^{m_{1}, n_{1}}\right) L_{\mathfrak{n s}}\left(c_{p, q}, h_{p, q}^{m_{2}, n_{2}}\right)
\end{array}\right) .
$$

Then we have:

1. For any $w_{(1)} \in L_{\mathfrak{n s}}\left(c_{p, q}, h_{p, q}^{m_{1}, n_{1}}\right)$ and $w_{(2)} \in L_{\mathfrak{n} \mathfrak{s}}\left(c_{p, q}, h_{p, q}^{m_{2}, n_{2}}\right)$,

$$
\mathcal{Y}\left(w_{(1)}, x\right) w_{(2)} \in x^{h_{p, q}^{m_{3}, n_{3}}-h_{p, q}^{m_{1}, n_{1}}-h_{p, q}^{m_{2}, n_{2}}} L_{\mathfrak{n s}}\left(c_{p, q}, h_{p, q}^{m_{3}, n_{3}}\right)\left(\left(x^{1 / 2}\right)\right)
$$


2. The map $\mathcal{Y}$ is uniquely determined by the maps

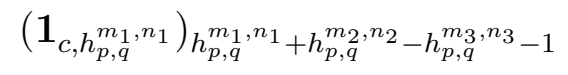

and

$$
\left(G(-1 / 2) \mathbf{1}_{c, h_{p, q}}^{m_{1}, n_{1}}\right)_{h_{p, q}^{m_{1}, n_{1}}+h_{p, q}^{m_{2}, n_{2}}-h_{p, q}^{m_{3}, n_{3}}-1 / 2}
$$

from $W_{2}$ to $W_{3}$ (recalling that $\mathbf{1}_{c, h_{p, q}^{m_{1}, n_{1}}}$ is the lowest weight vector in $\left.L_{\mathfrak{n} \mathfrak{s}}\left(c_{p, q}, h_{p, q}^{m_{1}, n_{1}}\right)\right)$, that is,

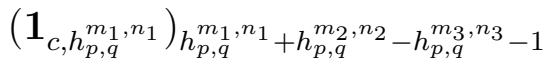

$$
\begin{aligned}
& =\left(G(-1 / 2) \mathbf{1}_{\left.c, h_{p, q}^{m_{1}, n_{1}}\right)}\right)_{h_{p, q}^{m_{1}, n_{1}}+h_{p, q}^{m_{2}, n_{2}}-h_{p, q}^{m_{3}, n_{3}}-1 / 2} \\
& =0
\end{aligned}
$$

implies $\mathcal{Y}=0$.

3. The space

$$
\mathcal{V}_{L_{\mathfrak{n} \mathfrak{S}}\left(c_{p, q}, h_{p, q}^{m_{1}, n_{1}}\right) L_{\mathfrak{n} \mathfrak{S}}\left(c_{p, q}, h_{p, q}^{m_{2}, n_{2}}\right)}^{L_{\mathrm{m}}\left(c_{p, q}, h_{3}^{m_{3}, n_{3}}\right)}
$$

is at most 2-dimensional.

Proof. Conclusion 1 is clear since the three modules are irreducible.

We prove Conclusion 2 now. For convenience, we denote $h_{p, q}^{m_{3}, n_{3}}-h_{p, q}^{m_{1}, n_{1}}-$ $h_{p, q}^{m_{2}, n_{2}}$ by $\Delta$ and $\mathbf{1}_{c, h_{p, q}^{m_{i}, n_{i}}}$ for $i=1,2,3$ by $w_{(i)}$, respectively. Suppose that

$$
\left(w_{(1)}\right)_{\Delta-1}=\left(G(-1 / 2) w_{(1)}\right)_{\Delta-1 / 2}=0
$$

but $\mathcal{Y} \neq 0$.

We need the following commutator formulas which are consequences of the Jacobi identity: For $n \in \mathbb{Z}, m \in \Delta+\mathbb{Z} / 2$,

$$
\begin{aligned}
& {\left[L(n),\left(w_{(1)}\right)_{m}\right]} \\
& \quad=\left(-m-n-1+(n+1) h_{p, q}^{m_{1}, n_{1}}\right)\left(w_{(1)}\right)_{m+n}, \\
& \quad\left[L(n),\left(G(-1 / 2) w_{(1)}\right)_{m}\right] \\
& \quad=\left(-m-n-1+(n+1) h_{p, q}^{m_{1}, n_{1}}\right)\left(G(-1 / 2) w_{(1)}\right)_{m+n}, \\
& \quad\left[G(n+1 / 2),\left(w_{(1)}\right)_{m}\right] \\
& \quad=\left(G(-1 / 2) w_{(1)}\right)_{m+n+1}, \\
& {\left[G(n+1 / 2),\left(G(-1 / 2) w_{(1)}\right)_{m}\right]} \\
& \quad=\left(-m-n-1 / 2-2 h_{p, q}^{m_{1}, n_{1}}\right)\left(w_{(1)}\right)_{m+n} .
\end{aligned}
$$


We claim that either

$$
\mathcal{Y}\left(w_{(1)}, x\right) w_{(2)} \neq 0
$$

or

$$
\mathcal{Y}\left(G(-1 / 2) w_{(1)}, x\right) w_{(2)} \neq 0 .
$$

In fact, if

$$
\mathcal{Y}\left(w_{(1)}, x\right) w_{(2)}=\mathcal{Y}\left(G(-1 / 2) w_{(1)}, x\right) w_{(2)}=0,
$$

then by the commutator formulas above, $\mathcal{Y}\left(w_{(1)}, x\right)=0$ which is a contradiction since $L_{\mathfrak{n s}}\left(c_{p, q}, h_{p, q}^{m_{1}, n_{1}}\right)$ is an irreducible $\mathfrak{n} \mathfrak{s}$-module and $\mathcal{Y} \neq 0$.

Let

$$
k=\max \left\{m \in \Delta+\mathbb{Z} / 2 \mid\left(w_{(1)}\right)_{m} w_{(2)} \neq 0 \text { or }\left(G(-1 / 2) w_{(1)}\right)_{m+1 / 2} w_{(2)} \neq 0\right\} .
$$

If

$$
\left(w_{(1)}\right)_{k} w_{(2)} \neq 0,
$$

then from the commutator formulas above, it follows that

$$
L(n)\left(w_{(1)}\right)_{k} w_{(2)}=\left(-k-n-1+(n+1) h_{p, q}^{m_{1}, n_{1}}\right)\left(w_{(1)}\right)_{k+n} w_{(2)}=0
$$

for $n \geq 1$ and

$$
G(n+1 / 2)\left(w_{(1)}\right)_{k} w_{(2)}=\left(G(-1 / 2) w_{(1)}\right)_{k+n+1} w_{(2)}=0
$$

for $n \geq 0$. Thus $\left(w_{(1)}\right)_{k} w_{(2)}$ is a multiple of the lowest weight vector $w_{(3)}$. Then

$$
h_{p, q}^{m_{3}, n_{3}}=\mathrm{wt}\left(\left(w_{(1)}\right)_{k} w_{(2)}\right)=h_{p, q}^{m_{1}, n_{1}}-k-1+h_{p, q}^{m_{2}, n_{2}} .
$$

It follows that $k=\Delta-1$. So $\left(w_{(1)}\right)_{k} w_{(2)}=0$, a contradiction.

If

$$
\left(G(-1 / 2) w_{(1)}\right)_{k+1 / 2} w_{(2)} \neq 0,
$$

then

$$
\begin{aligned}
& L(n)\left(G(-1 / 2) w_{(1)}\right)_{k+1 / 2} w_{(2)} \\
& \quad=\left(-k-1 / 2-n-1+(n+1) h_{p, q}^{m_{1}, n_{1}}\right)\left(G(-1 / 2) w_{(1)}\right)_{k+1 / 2+n} \\
& \quad=0
\end{aligned}
$$

for $n \geq 1$ and

$$
\begin{aligned}
G( & n+1 / 2)\left(G(-1 / 2) w_{(1)}\right)_{k+1 / 2} w_{(2)} \\
\quad & =\left(-k-n-1-2 h_{p, q}^{m_{1}, n_{1}}\left(w_{(1)}\right)_{k+n+1}\right. \\
& =0
\end{aligned}
$$


for $n \geq 0$. Thus $\left(G(-1 / 2) w_{(1)}\right)_{k+1 / 2} w_{(2)}$ is a highest weight vector and therefore

$$
h_{p, q}^{m_{3}, n_{3}}=\mathrm{wt}\left(G(-1 / 2) w_{(1)}\right)_{k+1 / 2} w_{(2)}=h_{p, q}^{m_{1}, n_{1}}-k-1+h_{p, q}^{m_{2}, n_{2}} .
$$

It follows that $k=\Delta-1$. So $\left(G(-1 / 2) w_{(1)}\right)_{k+1 / 2} w_{(2)}=0$, a contradiction.

Conclusion 3 follows immediately from Conclusion 2 .

Combining Theorem 2.1 and the third conclusion of Proposition 2.2, we obtain:

Corollary 2.3 The minimal $N=1$ superconformal vertex operator superalgebras are rational in the sense of [HL1], that is, the following three conditions are satisfied:

1. Every module for such an algebra is completely reducible.

2. There are only finitely many inequivalent irreducible modules for such an algebra.

3. The fusion rules among any three (irreducible) modules are finite.

Remark 2.4 In [Z], Zhu introduced a weaker notion of module for a vertex operator algebra and a notion of rational vertex operator algebra. Zhu's notion of rational vertex operator algebra is different from the notion of rational vertex operator algebra in [HL1 because Zhu's notion requires a stronger completely reducibility result for modules in his sense. The complete reducibility result and the classification of irreducible modules proved in $\mathrm{Ad}$. together with the third conclusion of Proposition 2.2 gives only the rationality in the sense of HL1.

We also have:

Proposition 2.5 Any finitely-generated lower truncated generalized $L_{\mathfrak{n} \mathfrak{s}}\left(c_{p, q}, 0\right)$ module $W$ is an ordinary module.

Proof. Suppose that $W$ is generated by a single vector $w \in W$. Then by the Poincaré-Birkhoff-Witt theorem and the lower truncation condition, every homogeneous subspace of $U(\mathfrak{n s}) w$ is finite-dimensional, proving the result. 
Let $n$ be a positive integer, $\left(p_{i}, q_{i}\right), i=1, \ldots, n, n$ pairs of integers larger than 1 such that $p_{i}-q_{i} \in 2 \mathbb{Z}$ and $\left(p_{i}-q_{i}\right) / 2$ and $q_{i}$ are relatively prime to each other, and let $V=L_{\mathfrak{n s}}\left(c_{p_{1}, q_{1}}, 0\right) \otimes \cdots \otimes L_{\mathfrak{n s}}\left(c_{p_{n}, q_{n}}, 0\right)$. From the trivial generalizations of the results proved in [FHD] and [DMZ] to vertex operator superalgebras, $V$ is a rational $N=1$ superconformal vertex operator superalgebra, a set of representatives of equivalence classes of irreducible modules for $V$ can be listed explicitly and the fusion rules for $V$ are finite and can be calculated easily.

We introduce a class of $N=1$ superconformal vertex operator vertex operator superalgebras:

Definition 2.6 Let $n$ be a positive integer, $\left(p_{i}, q_{i}\right), i=1, \ldots, n, n$ pairs of integers larger than 1 such that $p_{i}-q_{i} \in 2 \mathbb{Z}$ and $\left(p_{i}-q_{i}\right) / 2$ and $q_{i}$ are relatively prime to each other. An $N=1$ superconformal vertex operator vertex operator superalgebra $V$ is said to be in the class $\mathcal{C}_{p_{1}, q_{1} ; \ldots ; p_{n}, q_{n}}$ if $V$ has a vertex operator subalgebra isomorphic to $L_{\mathfrak{n s}}\left(c_{p_{1}, q_{1}}, 0\right) \otimes \cdots \otimes L_{\mathfrak{n s}}\left(c_{p_{m}, q_{m}}, 0\right)$.

Proposition 2.7 Let $V$ be an $N=1$ superconformal vertex operator vertex operator superalgebra in the class $\mathcal{C}_{p_{1}, q_{1} ; \ldots ; p_{n}, q_{n}}$. Then any finitely-generated lower truncated generalized $V$-module $W$ is an ordinary module.

Proof. The proof is similar to the proof of Proposition 3.7 in [H2]. So here we only point out the main difference. As in [H2], we discuss only the case $n=2$. Similar to the proof of Proposition 3.7 in [H2], using the Jacobi identity, the formula $G(-1 / 2)^{2}=L(-1)$ and Theorem 4.7.4 of [EHD, we can reduce our proof in the case of $n=2$ to the finite-dimensionality of the space spanned by the elements of the form

$$
\begin{aligned}
& L\left(-m_{1}^{(1)}\right) \cdots L\left(-m_{e_{1}}^{(1)}\right) G\left(-a_{1}^{(1)}\right) \cdots G\left(-a_{r_{1}}^{(1)}\right)\left(L(-1)^{l_{1}} G(-1 / 2)^{k_{1}} u_{(j)}^{(1)}\right)_{j_{1}} \cdot \\
& \quad \cdot L\left(n_{1}^{(1)}\right) \cdots L\left(n_{f_{1}}^{(1)}\right) G\left(b_{1}^{(1)}\right) \cdots G\left(b_{s_{1}}^{(1)}\right) w_{(t)}^{1} \\
& \quad \otimes L\left(-m_{1}^{(2)}\right) \cdots L\left(-m_{e_{2}}^{(2)}\right) G\left(-a_{1}^{(2)}\right) \cdots G\left(-a_{r_{2}}^{(2)}\right) \cdot \\
& \quad \cdot\left(L(-1)^{l_{2}} G(-1 / 2)^{k_{2}} u_{(j)}^{(2)}\right)_{j_{2}} L\left(n_{1}^{(2)}\right) \cdots L\left(n_{f_{2}}^{(2)}\right) G\left(b_{1}^{(2)}\right) \cdots G\left(b_{s_{2}}^{(2)}\right) w_{(t)}^{2},
\end{aligned}
$$

for $m_{1}^{(1)}, \ldots, m_{e_{1}}^{(1)}, m_{1}^{(2)}, \ldots, m_{e_{2}}^{(2)}, n_{1}^{(1)}, \ldots, n_{f_{1}}^{(1)}, n_{1}^{(2)}, \ldots, n_{f_{2}}^{(2)}, a_{1}^{(1)}, \ldots, a_{r_{1}}^{(1)}$, $a_{1}^{(2)}, \ldots, a_{r_{2}}^{(2)}, b_{1}^{(1)}, \ldots, b_{s_{1}}^{(1)}, b_{1}^{(2)}, \ldots, b_{s_{2}}^{(2)} \in \mathbb{Z}_{+}, l_{1}, l_{2} \in \mathbb{N}, k_{1}, k_{2}=0,1, j_{1}, j_{2} \in$ 
$\mathbb{Q}, t=1, \ldots, c, j=1, \ldots, d$, where $u_{(j)}^{(i)}, j=1, \ldots, d, i=1,2$, are elements of $V$ such that the $L_{\mathfrak{n s}}\left(c_{p_{i}, q_{i}}, 0\right)$-submodules generated by them isomorphic to $L\left(c_{p, q}, h_{p_{i}, q_{i}}^{m_{j}, n_{j}}\right)$ with the images of $u_{(j)}^{(i)}, j=1, \ldots, d, i=1,2$, as the lowest weight vectors and such that $V$ is isomorphic to the direct sum of these submodules, and where $w_{(t)}^{(i)}, t=1, \ldots, c, i=1,2$, are homogeneous elements of some irreducible $L_{\mathfrak{n s}}\left(c_{p_{i}, q_{i}}, 0\right)$-modules. Using the $L(-1)$-derivative property for generalized modules, we see that elements of the form (2.6) are spanned by elements of the form (2.6) with $l_{1}=l_{2}=0$. There are only finitely many of elements of the form (2.6) with $l_{1}=l_{2}=0$ and of a fixed weight $s$ because $W$ is lower truncated. Thus the homogeneous subspaces of $W$ are finite dimensional. So $W$ is a $V$-module.

\section{Correlation functions and differential equa- tions of regular singular points}

In this section, we study products and iterates of intertwining operators for the minimal $N=1$ superconformal vertex operator superalgebras. The goal is to prove that these products and iterates satisfying the convergence and extension properties introduced in [H]]. The main tool is differential equations of regular singular points. Though the strategy of the proof is similar to that in [H2], there are subtle and nontrivial differences. We shall be brief on the parts of proofs which are similar to those in [H2] but give detailed discussions on the parts which are different.

Let $p, q$ be integers larger than 1 such that $p-q \in 2 \mathbb{Z}$ and $(p-q) / 2$ and $q$ are relatively prime to each other. Let $W_{i}, i=1, \ldots, 5$, be irreducible modules for the vertex operator algebra $L_{\mathrm{ns}}\left(c_{p, q}, 0\right)$ and $\mathcal{Y}_{1}$ and $\mathcal{Y}_{2}$ intertwining operators of type $\left(\begin{array}{c}W_{4} \\ W_{1} W_{5}\end{array}\right)$ and $\left(\begin{array}{c}W_{5} \\ W_{2} W_{3}\end{array}\right)$, respectively.

We first state a proposition which describes matrix coefficients of the products of $\mathcal{Y}_{1}$ and $\mathcal{Y}_{2}$ with odd variables. The proof is very easy and is omitted.

Proposition 3.1 Let $w_{(i)} \in W_{i}(i=1,2,3)$ and $w_{(4)}^{\prime} \in W_{4}^{\prime}$. Then

$$
\begin{aligned}
& \left\langle w_{(4)}^{\prime}, \mathcal{Y}_{1}\left(w_{(1)},\left(x_{1}, \varphi_{1}\right)\right) \mathcal{Y}_{2}\left(w_{(2)},\left(x_{2}, \varphi_{2}\right)\right) w_{(3)}\right\rangle \\
& \quad=Q_{0,0}\left(x_{1}, x_{2}\right)+\varphi_{1} Q_{0,1}\left(x_{1}, x_{2}\right)+\varphi_{2} Q_{1,0}\left(x_{1}, x_{2}\right)+\varphi_{1} \varphi_{2} Q_{1,1}\left(x_{1}, x_{2}\right)
\end{aligned}
$$


where $Q_{k, l}\left(x_{1}, x_{2}\right)=x_{1}^{t_{1}-k / 2} x_{2}^{t_{2}-l / 2} R_{k, l}\left(x_{2}^{1 / 2} / x_{1}^{1 / 2}\right)$, and $t_{1}, t_{2} \in \mathbb{Q}, R_{k, l}(x) \in$ $\mathbb{C}[[x]](k, l=0,1)$.

We first prove the following theorem:

Theorem 3.2 Let $w_{(i)} \in W_{i}(i=1,2,3)$ and $w_{(4)}^{\prime} \in W_{4}^{\prime}$ be the lowest weight vectors. Then we have:

1. The series

$$
\left.\left\langle w_{(4)}^{\prime}, \mathcal{Y}_{1}\left(w_{(1)},\left(x_{1}, \varphi_{1}\right)\right) \mathcal{Y}_{2}\left(w_{(2)},\left(x_{2}, \varphi_{2}\right)\right) w_{(3)}\right\rangle\right|_{x_{j}^{n}=e^{n \log z_{j}, j=1,2}}
$$

is convergent to a multivalued (Grassman) analytic function in the region $\left|z_{1}\right|>\left|z_{2}\right|>0$ for any choice of $\log z_{1}$ and $\log z_{2}$.

2. For each $k, l=0,1$ there exist $m_{k, l} \in \mathbb{N}$, analytic functions $f_{i}^{k, l}(z)$ in the region $|z|<1$ and $s_{i}^{k, l}, r_{i}^{k, l} \in \mathbb{Q}$ for $i=1, \ldots, m_{k, l}$, such that $\left.Q_{k, l}\left(x_{1}, x_{2}\right)\right|_{x_{j}^{n}=e^{n \log z_{j}, j=1,2}}$ can be analytically extended to a multivalued analytic functions of the form

$$
\sum_{i=1}^{m_{k, l}} z_{2}^{s_{i}^{k, l}}\left(z_{1}-z_{2}\right)^{r_{i}^{k, l}} f_{i}^{k, l}\left(\frac{z_{1}-z_{2}}{z_{2}}\right)
$$

when $\left|z_{2}\right|>\left|z_{1}-z_{2}\right|>0$.

Proof. From Proposition 3.1, we see that to prove the Conclusion 1, it is enough to prove that the series $\left.Q_{k, l}\left(x_{1} . x_{2}\right)\right|_{x_{i}^{n}=e^{n \log z_{i}, i=1,2}}, k, l=0,1$, are absolutely convergent in the region $\left|z_{1}\right|>\left|z_{2}\right|>0$.

We need the following structural result about singular vectors obtained by Astashkevich in As]:

Lemma 3.3 Let $M_{\mathfrak{n s}}\left(c_{p . q}, h\right)$ be a Verma module for $\mathfrak{n s}$ and $w \in M_{\mathfrak{n s}}\left(c_{p . q}, h\right)$ a singular vector of weight $n+h(n \in \mathbb{N} / 2)$. Then

$$
w=a\left(G(-1 / 2)^{2 n}+\cdots\right) \mathbf{1}_{c_{p, q}, h},
$$

where $a$ is a nonzero complex number and $\cdots$ denotes a sum of monomials different from $G(-1 / 2)^{2 n}$. 
Since the irreducible module $W_{3}$ is a quotient of a Verma module by the submodule generated by two singular vectors, we can find nonzero $P \in$ $U\left(\mathfrak{n s}^{-}\right)$such that $P w_{(3)}=0$ in $W_{3}$. Moreover, we can choose such a $P$ to be even because otherwise we can compose it with $G(-1 / 2)$. By Lemma 3.3, we can alway normalize $P$ such that $P=G(-1 / 2)^{2 n}+\cdots$ where $\cdots$ denotes a sum of monomials different from $G(-1 / 2)^{2 n}$.

Now we prove Conclusion 1 for $Q_{0,1}$ and $Q_{1,0}$. From $P w_{(3)}=0$ and the commutator formula between $G(n+1 / 2), L(n), n \in \mathbb{Z}$, and intertwining operators, we obtain a system of differential equations

$$
\begin{aligned}
0 & =\left\langle w_{(4)}^{\prime}, \mathcal{Y}_{1}\left(G(-1 / 2) w_{(1)}, z_{1}\right) \mathcal{Y}_{2}\left(w_{(2)}, z_{2}\right) P w_{(3)}\right\rangle \\
& =D_{1}\left(z_{1}, z_{2}\right) Q_{1,0}+D_{2}\left(z_{1}, z_{2}\right) Q_{0,1}, \\
0 & =\left\langle w_{(4)}^{\prime}, \mathcal{Y}_{1}\left(w_{(1)}, z_{1}\right) \mathcal{Y}_{2}\left(G(-1 / 2) w_{(2)}, z_{2}\right) P w_{(3)}\right\rangle \\
& =D_{3}\left(z_{1}, z_{2}\right) Q_{1,0}+D_{4}\left(z_{1}, z_{2}\right) Q_{0,1}
\end{aligned}
$$

for $Q_{1,0}$ and $Q_{0,1}$ of regular singular points with the only possible singular points $z_{1}, z_{2}=0, \infty$.

Since $P=G(-1 / 2)^{2 n}+\cdots$ where $\cdots$ denotes a sum of monomials different from $G(-1 / 2)^{2 n}$, we have

$$
\begin{aligned}
D_{1} & =\left(\partial_{z_{1}}+\partial_{z_{2}}\right)^{n}+\sum_{i+j \leq n-1} f_{i, j}\left(z_{1}, z_{2}\right) \partial_{z_{1}}^{i} \partial_{z_{2}}^{j}, \\
D_{2} & =\sum_{i+j \leq n-1} g_{i, j}\left(z_{1}, z_{2}\right) \partial_{z_{1}}^{i} \partial_{z_{2}}^{j}, \\
D_{3} & =\sum_{i+j \leq n-1} k_{i, j}\left(z_{1}, z_{2}\right) \partial_{z_{1}}^{i} \partial_{z_{2}}^{j}, \\
D_{4} & =\left(\partial_{z_{1}}+\partial_{z_{2}}\right)^{n}+\sum_{i+j \leq n-1} h_{i, j}\left(z_{1}, z_{2}\right) \partial_{z_{1}}^{i} \partial_{z_{2}}^{j}
\end{aligned}
$$

for certain meromorphic functions $f_{i, j}, g_{i, j}, h_{i, j}$ and $k_{i, j}$.

Because of the structure of $Q_{1,0}$ and $Q_{0,1}$, we can reduce the system (3.4)(3.5) to a system

$$
\begin{aligned}
& \hat{D}_{1}(z) R_{1,0}(z)+\hat{D}_{2}(z) R_{0,1}(z)=0 \\
& \hat{D}_{3}(z) R_{1,0}(z)+\hat{D}_{4}(z) R_{0,1}(z)=0
\end{aligned}
$$

with analytic coefficients in one variable $z=z_{2} / z_{1}$ in the region $0<|z|<1$ (there might be a singularity at $z=0$ ) for $R_{1,0}$ and $R_{0,1}$, where $\hat{D}_{i}(z)$, 
$i=1,2,3,4$, have the following form

$$
\begin{aligned}
\hat{D}_{1}(z) & =\partial_{z}^{n}+\sum_{i \leq n-1} f_{i}(z) \partial_{z}^{i}, \\
\hat{D}_{2}(z) & =\sum_{i \leq n-1} g_{i}(z) \partial_{z}^{i}, \\
\hat{D}_{4}(z) & =\partial_{z}^{n}+\sum_{i \leq n-1} h_{i}(z) \partial_{z}^{i}, \\
\hat{D}_{3}(z) & =\sum_{i \leq n-1} k_{i}(z) \partial_{z}^{i}
\end{aligned}
$$

where $f_{i}, g_{i}, h_{i}, k_{i}$ are analytic functions with possible poles at $z=0$. This (in general higher order) linear system of equations can be further reduced to a linear system of equations of regular singular points of first order. (More precisely we introduce new unknowns $A_{i}=R_{1,0}^{(i)}$ and $B_{i}=R_{0,1}^{(i)}, i=0, \ldots, n-$ 1. Then we have a first-order linear system of $2 n$ equations for $A_{i}$ 's and $B_{i}$ 's, $i=0, \ldots, n-1$.) From the theory of differential equations of regular singular points, it follows that $R_{1,0}(z)$ and $R_{0,1}(z)$ are absolutely convergent in the region $|z|<1$. Thus $Q_{1,0}$ and $Q_{0,1}$ are absolutely convergent to analytic functions in the region $\left|z_{1}\right|>\left|z_{2}\right|>0$.

Now we would like to extend $Q_{1,0}$ and $Q_{0,1}$ analytically to functions of the form (3.2) in the region $\left|z_{2}\right|>\left|z_{1}-z_{2}\right|>0$. Let $Q$ be a nonzero element of $U\left(\mathfrak{n s}^{-}\right)$such that $Q w_{(2)}=0$ in $W_{2}$. Similar to $P$, we can choose $Q$ to be even and we can normalize $Q$ such that $Q=G(-1 / 2)^{2 m}+\cdots$ where $\cdots$ denotes a sum of monomials different from $G(-1 / 2)^{2 m}$. From $Q w_{(2)}=0$, the commutator formulas between $G(n+1 / 2)$ and $L(n), n \in \mathbb{Z}$, and the Jacobi identity for intertwining operators, we have

$$
\begin{aligned}
0 & =\left\langle w_{(4)}^{\prime}, \mathcal{Y}_{1}\left(G(-1 / 2) w_{(1)}, z_{1}\right) \mathcal{Y}_{2}\left(Q w_{(2)}, z_{2}\right) w_{(3)}\right\rangle \\
& =D_{1}^{\prime}\left(z_{1}, z_{2}\right) Q_{1,0}+D_{2}^{\prime}\left(z_{1}, z_{2}\right) Q_{0,1} \\
0 & =\left\langle w_{(4)}^{\prime}, \mathcal{Y}_{1}\left(w_{(1)}, z_{1}\right) \mathcal{Y}_{2}\left(G(-1 / 2) Q w_{(2)}, z_{2}\right) P w_{(3)}\right\rangle \\
& =D_{3}^{\prime}\left(z_{1}, z_{2}\right) Q_{1,0}^{\prime}+D_{4}\left(z_{1}, z_{2}\right) Q_{0,1}
\end{aligned}
$$

for some differential operators $D_{1}^{\prime}, D_{2}^{\prime}, D_{3}^{\prime}$ and $D_{4}^{\prime}$.

From the formulas used to derive (3.6) -(3.7) and from $Q=G(-1 / 2)^{2 m}+$ ‥ where $\cdots$ denotes a sum of monomials different from $G(-1 / 2)^{2 m}$, it is easy to see that (3.6)-(3.7) is a system of equations of regular singular points 
with the only possible singular points $z_{2}, z_{1}-z_{2}=0, \infty$. Since (3.4)-(3.5) is a system of equations of regular singular points with the only possible singular points $z_{1}=z_{2}=0, \infty$, the equations (3.4), (3.5) and the equations (3.6), (3.7) are independent. Thus we obtain a system (3.4)-(3.7) of equations of regular singular points with the only possible singularities $z_{1}, z_{2}=0, \infty$ and $z_{1}=z_{2}$. This system is consistent because it has a formal series solution $\left(Q_{1,0}, Q_{0,1}\right)$. Since we have proved that $Q_{1,0}$ and $Q_{0,1}$ are absolutely convergent in the region $\left|z_{1}\right|>\left|z_{2}\right|>0$ and since the intersection $\left|z_{1}\right|>\left|z_{2}\right|>\left|z_{1}-z_{2}\right|>0$ of the regions $\left|z_{1}\right|>\left|z_{2}\right|>0$ and $\left|z_{2}\right|>\left|z_{1}-z_{2}\right|>0$ are nonempty, we can use the sum of $Q_{1,0}$ and $Q_{0,1}$ at a particular point in the intersection $\left|z_{1}\right|>\left|z_{2}\right|>\left|z_{1}-z_{2}\right|>0$ as initial conditions for the system (3.4)-(3.7). Then by the theory of differential equations of regular singular points, this initial value problem has a unique solution of the form (3.2) in the region $\left|z_{2}\right|>\left|z_{1}-z_{2}\right|>0$ (without logarithm terms because the analytic extension of the solution in the region $\left|z_{1}\right|>\left|z_{2}\right|>0$ does not have logarithm terms). So $Q_{0,1}$ and $Q_{1,0}$ can be analytically extended to analytic functions of the form (3.2) in the region $\left|z_{2}\right|>\left|z_{1}-z_{2}\right|>0$.

We have to do the same for $Q_{0,0}$ and $Q_{1,1}$. By using the commutator formulas between $G(n+1 / 2), L(n), n \in \mathbb{Z}$, and the intertwining operators, we have

$$
\begin{aligned}
0 & =\left\langle w_{(4)}^{\prime}, \mathcal{Y}_{1}\left(w_{(1)}, z_{1}\right) \mathcal{Y}_{2}\left(w_{(2)}, z_{2}\right) P w_{(3)}\right\rangle \\
& =D_{5}\left(z_{1}, z_{2}\right) Q_{0,0}+D_{6}\left(z_{1}, z_{2}\right) Q_{1,1} \\
0 & =\left\langle w_{(4)}^{\prime}, \mathcal{Y}_{1}\left(G(-1 / 2) w_{(1)}, z_{1}\right) \mathcal{Y}_{2}\left(G(-1 / 2) w_{(2)}, z_{2}\right) P w_{(3)}\right\rangle \\
& =D_{7}\left(z_{1}, z_{2}\right) Q_{0,0}+D_{8}\left(z_{1}, z_{2}\right) Q_{1,1} \\
0 & =\left\langle w_{(4)}^{\prime}, \mathcal{Y}_{1}\left(w_{(1)}, z_{1}\right) \mathcal{Y}_{2}\left(Q w_{(2)}, z_{2}\right) w_{(3)}\right\rangle \\
& =D_{5}^{\prime}\left(z_{1}, z_{2}\right) Q_{0,0}+D_{6}^{\prime}\left(z_{1}, z_{2}\right) Q_{1,1}, \\
0 & =\left\langle w_{(4)}^{\prime}, \mathcal{Y}_{1}\left(G(-1 / 2) w_{(1)}, z_{1}\right) \mathcal{Y}_{2}\left(G(-1 / 2) Q w_{(2)}, z_{2}\right) w_{(3)}\right\rangle \\
& =D_{7}^{\prime}\left(z_{1}, z_{2}\right) Q_{0,0}+D_{8}^{\prime}\left(z_{1}, z_{2}\right) Q_{1,1}
\end{aligned}
$$

for some differential operators $D_{5}, D_{6}, D_{7}, D_{8}, D_{5}^{\prime}, D_{6}^{\prime}, D_{7}^{\prime}$ and $D_{8}^{\prime}$. From the structures of $P$ and $Q$, we see that these equations form a system of equations of regular singular points with the only possible singular points $z_{1}, z_{2}=0, \infty$ and $z_{1}-z_{2}$. Using this system, we can prove the conclusions we need for $Q_{0,0}$ and $Q_{1,1}$. We omit the details since they are completely the same as those for $Q_{1,0}$ and $Q_{0,1}$.

The main goal of this section is the following more general result: 
Theorem 3.4 For any quadruple $\left\{w_{(1)}, w_{(2)}, w_{(2)}, w_{(4)}^{\prime}\right\}$ where $w_{(i)} \in W_{i}, i=$ $1,2,3$, and $w_{(4)}^{\prime} \in W_{4}^{\prime}$ are homogeneous vectors, the statement of Theorem 3.9 is true. Moreover there is an integer $N$ depending only on $\mathcal{Y}_{1}$ and $\mathcal{Y}_{1}$ such that

$$
\text { wt } w_{(1)}+\text { wt } w_{(2)}+s_{i}^{k, l}>N
$$

for all homogeneous elements $w_{(1)} \in W_{1}, w_{(2)} \in W_{2}, i=1, \ldots, m_{k, l}$ and $k, l=0,1$. That is, in the terminology introduced in [H1], the products or the iterates of the intertwining operators for $L_{\mathfrak{n s}}\left(c_{p, q}, 0\right)$ have the convergence and extension property.

Proof. We define the weight of a quadruple $\left\{w_{(1)}, w_{(2)}, w_{(2)}, w_{(4)}^{\prime}\right\}$ to be $r=$ wt $w_{(1)}+$ wt $w_{(2)}+$ wt $w_{(3)}+$ wt $w_{(4)}^{\prime}$. We shall use induction on the weight $r$. Theorem 3.2 gives the result in the case of the smallest $r$.

We have the following commutator formulas (which follow from the Jacobi identity):

$$
\begin{aligned}
& {\left[Y\left(\omega, x_{1}\right), \mathcal{Y}\left(w_{(1)},\left(x_{2}, \varphi\right)\right)\right]} \\
& =\left(x_{1}^{-1} \delta\left(x_{2} / x_{1}\right) \partial_{x_{2}}+x_{1}^{-1} \partial_{x_{2}} \delta\left(x_{2} / x_{1}\right)\left(\left(\operatorname{wt} w_{(1)}\right)+1 / 2 \varphi \partial_{\varphi}\right)\right) \mathcal{Y}\left(w_{(1)},\left(x_{2}, \varphi\right)\right) \\
& \quad+\cdots \\
& \left.\quad \cdots(-n), \mathcal{Y}\left(w_{(1)},\left(x_{2}, \varphi\right)\right)\right] \\
& =\left(x_{2}^{-n+1} \partial_{x_{2}}+(1-n) x_{2}^{-n}\left(\left(\operatorname{wt} w_{(1)}\right)+1 / 2 \varphi \partial_{\varphi}\right)\right) \mathcal{Y}\left(w_{(1)},\left(x_{2}, \varphi\right)\right) \\
& \quad+\cdots \\
& \quad \cdots Y \\
& \left.\quad\left(\tau, x_{1}\right), \mathcal{Y}\left(w_{(1)},\left(x_{2}, \varphi\right)\right)\right] \\
& \quad\left(x_{1}^{-1} \delta\left(x_{2} / x_{1}\right)\left(\partial_{\varphi}-\varphi \partial_{x_{2}}\right)-2 x_{1}^{-1} \partial_{x_{2}} \delta\left(x_{2} / x_{1}\right)\left(\operatorname{wt} w_{(1)}\right) \varphi\right) \mathcal{Y}\left(w_{(1)},\left(x_{2}, \varphi\right)\right) \\
& \quad+\cdots \\
& {\left[G(-n-1 / 2), \mathcal{Y}\left(w_{(1)},\left(x_{2}, \varphi\right)\right)\right]} \\
& \quad=\left(x_{2}^{-n}\left(\partial_{\varphi}-\varphi \partial_{x_{2}}\right)+2 n x_{2}^{-n-1}\left(\operatorname{wt} w_{(1)}\right) \varphi\right) \mathcal{Y}\left(w_{(1)},\left(x_{2}, \varphi\right)\right)+\cdots
\end{aligned}
$$

where we write $\cdots$ for terms associated to elements whose weights are less than the weight of $w_{(1)}$.

To prove the theorem it is enough to show that if the statement is true for the quadruple $\left\{w_{(1)}, w_{(2)}, w_{(3)}, w_{(4)}^{\prime}\right\}$, then it is also true for the quadruples

$$
\begin{aligned}
& \left\{G(-n-1 / 2) w_{(1)}, w_{(2)}, w_{(3)}, w_{(4)}^{\prime}\right\},\left\{L(-n) w_{(1)}, w_{(2)}, w_{(3)}, w_{(4)}^{\prime}\right\}, \\
& \left\{w_{(1)}, G(-n-1 / 2) w_{(2)}, w_{(3)}, w_{(4)}^{\prime}\right\},\left\{w_{(1)}, L(-n) w_{(2)}, w_{(3)}, w_{(4)}^{\prime}\right\},
\end{aligned}
$$




$$
\begin{aligned}
& \left\{w_{(1)}, w_{(2)}, G(-n-1 / 2) w_{(3)}, w_{(4)}^{\prime}\right\},\left\{w_{(1)}, w_{(2)}, L(-n) w_{(3)}, w_{(4)}^{\prime}\right\} \\
& \left\{w_{(1)}, w_{(2)}, w_{(3)}, G(-n-1 / 2) w_{(4)}^{\prime}\right\},\left\{w_{(1)}, w_{(2)}, w_{(3)}, L(-n) w_{(4)}^{\prime}\right\}
\end{aligned}
$$

for every $n \in \mathbb{N}$. We shall prove the statement only for the quadruples

$$
\left\{w_{(1)}, w_{(2)}, G(-n-1 / 2) w_{(3)}, w_{(4)}^{\prime}\right\},\left\{w_{(1)}, w_{(2)}, L(-n) w_{(3)}, w_{(4)}^{\prime}\right\}
$$

for the others, the proofs are similar.

From (3.9)-(3.12) we get

$$
\begin{aligned}
\left\langle w_{(4)}^{\prime}, \mathcal{Y}_{1}\left(w_{(1)},\left(x_{1}, \varphi_{1}\right)\right) \mathcal{Y}_{2}\left(w_{(2)},\left(x_{2}, \varphi_{2}\right)\right) L(-n) w_{(3)}\right\rangle & \\
=- & \left(x_{1}^{-n+1} \partial_{x_{1}}+(1-n) x_{1}^{-n}\left(\left(\operatorname{wt} w_{(1)}\right)+1 / 2 \varphi_{1} \partial_{\varphi_{1}}\right)+x_{2}^{-n+1} \partial_{x_{2}}\right. \\
+ & (1-n) x_{2}^{-n}\left(\left(\text { wt } w_{(2)}\right)+1 / 2 \varphi_{2} \partial_{\varphi_{2}}\right) \cdot \\
& \cdot\left\langle w_{(4)}^{\prime}, \mathcal{Y}_{1}\left(w_{(1)},\left(x_{1}, \varphi_{1}\right)\right) \mathcal{Y}_{2}\left(w_{(2)},\left(x_{2}, \varphi_{2}\right)\right) w_{(3)}\right\rangle \\
+ & \left\langle L(n) w_{(4)}^{\prime}, \mathcal{Y}_{1}\left(w_{(1)},\left(x_{1}, \varphi_{1}\right)\right) \mathcal{Y}_{2}\left(w_{(2)},\left(x_{2}, \varphi_{2}\right)\right) w_{(3)}\right\rangle+\cdots
\end{aligned}
$$

and

$$
\begin{aligned}
&\left\langle w_{(4)}^{\prime}, \mathcal{Y}_{1}\left(w_{(1)},\left(x_{1}, \varphi_{1}\right)\right) \mathcal{Y}_{2}\left(w_{(2)},\left(x_{2}, \varphi_{2}\right)\right) G(-n-1 / 2) w_{(3)}\right\rangle \\
&=-\left(x_{1}^{-n}\left(\partial_{\varphi_{1}}-\varphi_{1} \partial_{x_{1}}\right)+2 n x_{1}^{-n-1}\left(\text { wt } w_{(1)}\right)\right) \varphi_{1}+x_{2}^{-n}\left(\partial_{\varphi_{2}}\right. \\
&-\left.\left.\varphi_{2} \partial_{x_{2}}\right)+2 n x_{2}^{-n-1}\left(\mathrm{wt} w_{(2)}\right) \varphi_{2}\right) \cdot \\
& \cdot\left\langle w_{(4)}^{\prime}, \mathcal{Y}_{1}\left(w_{(1)},\left(x_{1}, \varphi_{1}\right)\right) \mathcal{Y}_{2}\left(w_{(2)},\left(x_{2}, \varphi_{2}\right)\right) w_{(3)}\right\rangle \\
&+\left\langle G(n+1 / 2) w_{(4)}^{\prime}, \mathcal{Y}_{1}\left(w_{(1)},\left(x_{1}, \varphi_{1}\right)\right) \mathcal{Y}_{2}\left(w_{(2)},\left(x_{2}, \varphi_{2}\right)\right) w_{(3)}\right\rangle \\
&+\cdots,
\end{aligned}
$$

where $\cdots$ represents terms (certain derivatives of matrix coefficients) involving quadruples whose weights are less then $r$. Since the weights of quadruples $\left\{w_{(1)}, w_{(2)}, w_{(3)}, L(n) w_{(4)}^{\prime}\right\}$ and $\left\{w_{1}, w_{2}, w_{3}, G(n+1 / 2) w_{4}^{\prime}\right\}$ are $r-n$ and $r-n-1 / 2$ respectively we can apply induction.

We choose $N$ to be an integer such that when $w_{(1)}$ and $w_{(2)}$ are the lowest weight vectors, (3.8) holds. Then using the formulas above and induction, it is easy to see that (3.8) holds for all $w_{(1)} \in W_{1}$ and $w_{(2)} \in W_{2}$ (cf. [H2]).

Finally, we generalize Theorem 3.4 to vertex operator algebras in the class $\mathcal{C}_{p_{1}, q_{1} ; \ldots ; p_{n}, q_{n}}$ for $\left(p_{i}, q_{i}\right), i=1, \ldots, n, n$ pairs of integers larger than 1 such that $p_{i}-q_{i} \in 2 \mathbb{Z}$ and $\left(p_{i}-q_{i}\right) / 2$ and $q_{i}$ are relatively prime to each other. Since the proof of the following result is completely the same as the corresponding results in [H2] and [HL6], we omit the details here. 
Theorem 3.5 Let $V$ be a vertex operator algebra in the class $\mathcal{C}_{p_{1}, q_{1} ; \ldots ; p_{n}, q_{n}}$. Then the products and iterates of intertwining operators for $V$ have the convergence and extension property.

\section{Intertwining operator superalgebras and ver- tex tensor categories for $N=1$ minimal models}

In this section, $V$ is a vertex operator algebra in the class $\mathcal{C}_{p_{1}, q_{1} ; \ldots ; p_{n}, q_{n}}^{(1)}$ for $\left(p_{i}, q_{i}\right), i=1, \ldots, n, n$ pairs of integers larger than 1 such that $p_{i}-q_{i} \in 2 \mathbb{Z}$ and $\left(p_{i}-q_{i}\right) / 2$ and $q_{i}$ are relatively prime to each other. By Proposition 2.7 and Theorem 3.5, and Theorems 3.1 and 3.2 in [H2], which are in turn proved in [H2] using results in [HL1]- HL5] and [H1], we obtain the following:

Theorem 4.1 (associativity for intertwining operators) 1. For any $V$-modules $W_{0}, W_{1}, W_{2}, W_{3}$ and $W_{4}$, any intertwining operators $\mathcal{Y}_{1}$ and $\mathcal{Y}_{2}$ of types $\left(\begin{array}{c}W_{0} \\ W_{1} W_{4}\end{array}\right)$ and $\left(\begin{array}{c}W_{4} \\ W_{2} W_{3}\end{array}\right)$, respectively, and any choice of $\log z_{1}$ and $\log z_{2}$,

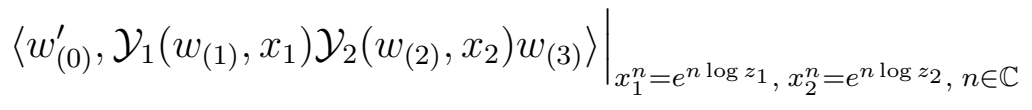

is absolutely convergent when $\left|z_{1}\right|>\left|z_{2}\right|>0$ for $w_{(0)}^{\prime} \in W_{0}^{\prime}, w_{(1)} \in W_{1}$, $w_{(2)} \in W_{2}$ and $w_{(3)} \in W_{3}$. For any modules $W_{0}, W_{1}, W_{2}, W_{3}$, and $W_{5}$ and any intertwining operators $\mathcal{Y}_{3}$ and $\mathcal{Y}_{4}$ of types $\left(\begin{array}{c}W_{5} \\ W_{1} W_{2}\end{array}\right)$ and $\left(\begin{array}{c}W_{0} \\ W_{5} W_{3}\end{array}\right)$, respectively, and any choice of $\log z_{2}$ and $\log \left(z_{1}-z_{2}\right)$,

$$
\left.\left\langle w_{(0)}^{\prime}, \mathcal{Y}_{4}\left(\mathcal{Y}_{3}\left(w_{(1)}, x_{0}\right) w_{(2)}, x_{2}\right) w_{(3)}\right\rangle\right|_{x_{0}^{n}=e^{n \log \left(z_{1}-z_{2}\right)}, x_{2}^{n}=e^{n \log z_{2}, n \in \mathbb{C}}}
$$

is absolutely convergent when $\left|z_{2}\right|>\left|z_{1}-z_{2}\right|>0$ for $w_{(0)}^{\prime} \in W_{0}^{\prime}$, $w_{(1)} \in W_{1}, w_{(2)} \in W_{2}$ and $w_{(3)} \in W_{3}$.

2. For any $V$-modules $W_{0}, W_{1}, W_{2}, W_{3}$ and $W_{4}$, any intertwining operators $\mathcal{Y}_{1}$ and $\mathcal{Y}_{2}$ of types $\left(\begin{array}{c}W_{0} \\ W_{1} W_{4}\end{array}\right)$ and $\left(\begin{array}{c}W_{4} \\ W_{2} W_{3}\end{array}\right)$, respectively, there exist a module $W_{5}$ and intertwining operators $\mathcal{Y}_{3}$ and $\mathcal{Y}_{4}$ of types $\left(\begin{array}{c}W_{5} \\ W_{1} W_{2}\end{array}\right)$ and $\left(\begin{array}{c}W_{0} \\ W_{5} W_{3}\end{array}\right)$, respectively, such that for any $z_{1}, z_{2} \in \mathbb{C}$ satisfying $\left|z_{1}\right|>$ 
$\left|z_{2}\right|>\left|z_{1}-z_{2}\right|>0$ and for any $w_{(0)}^{\prime} \in W_{0}^{\prime}, w_{(1)} \in W_{1}, w_{(2)} \in W_{2}$ and $w_{(3)} \in W_{3}$,

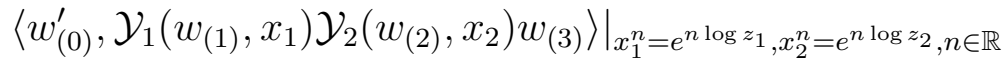

$$
\begin{aligned}
& \left.=\left\langle w_{(0)}^{\prime}, \mathcal{Y}_{4}\left(\mathcal{Y}_{3}\left(w_{(1)}, x_{0}\right) w_{(2)}, x_{2}\right) w_{(3)}\right\rangle\right\rangle\left.\right|_{x_{0}^{n}=e^{n \log \left(z_{1}-z_{2}\right)}, x_{2}^{n}=e^{n \log z_{2}, n \in \mathbb{R}}},
\end{aligned}
$$

where $\log z_{1}=\left|z_{1}\right|+i \arg z_{1}, \log z_{2}=\left|z_{2}\right|+i \arg z_{2}$ and $\log \left(z_{1}-z_{2}\right)=$ $\left|z_{1}-z_{2}\right|+i \arg \left(z_{1}-z_{2}\right)$ are the values of the logarithms of $z_{1}, z_{2}$ and $z_{1}-z_{2}$ such that $0 \leq \arg z_{1}, \arg z_{2}, \arg \left(z_{1}-z_{2}\right) \leq 2 \pi$.

3. For any modules $W_{0}, W_{1}, W_{2}, W_{3}$, and $W_{5}$, any intertwining operators $\mathcal{Y}_{3}$ and $\mathcal{Y}_{4}$ of types $\left(\begin{array}{c}W_{5} \\ W_{1} W_{2}\end{array}\right)$ and $\left(\begin{array}{c}W_{0} \\ W_{5} W_{3}\end{array}\right)$, respectively, there exist a module $W_{4}$ and intertwining operators $\mathcal{Y}_{1}$ and $\mathcal{Y}_{2}$ of types $\left(\begin{array}{c}W_{0} \\ W_{1} W_{4}\end{array}\right)$ and $\left(\begin{array}{c}W_{4} \\ W_{2} W_{3}\end{array}\right)$, respectively, such that for any $z_{1}, z_{2} \in \mathbb{C}$ satisfying $\left|z_{1}\right|>\left|z_{2}\right|>\mid z_{1}-$ $z_{2} \mid>0$ and for any $w_{(0)}^{\prime} \in W_{0}^{\prime}, w_{(1)} \in W_{1}, w_{(2)} \in W_{2}$ and $w_{(3)} \in W_{3}$, the equality (4.1) holds.

Theorem 4.2 (commutativity for intertwining operators) For any $V$ modules $W_{0}, W_{1}, W_{2}, W_{3}$ and $W_{4}$ and any intertwining operators $\mathcal{Y}_{1}$ and $\mathcal{Y}_{2}$ of types $\left(\begin{array}{c}W_{0} \\ W_{1} W_{4}\end{array}\right)$ and $\left(\begin{array}{c}W_{4} \\ W_{2} W_{3}\end{array}\right)$, respectively, there exist a module $W_{5}$ and intertwining operators $\mathcal{Y}_{3}$ and $\mathcal{Y}_{4}$ of types $\left(\begin{array}{c}W_{0} \\ W_{2} W_{5}\end{array}\right)$ and $\left(\begin{array}{c}W_{5} \\ W_{1} W_{3}\end{array}\right)$, respectively, such that for any homogeneous $w_{(0)}^{\prime} \in W_{0}^{\prime}, w_{(1)} \in W_{1}, w_{(2)} \in W_{2}$ and $w_{(3)} \in W_{3}$, the multivalued analytic function

$$
\left.\left\langle w_{(0)}^{\prime}, \mathcal{Y}_{1}\left(w_{(1)}, x_{1}\right) \mathcal{Y}_{2}\left(w_{(2)}, x_{2}\right) w_{(3)}\right\rangle\right|_{x_{1}=z_{1}, x_{2}=z_{2}}
$$

of $z_{1}$ and $z_{2}$ in the region $\left|z_{1}\right|>\left|z_{2}\right|>0$ and the multivalued analytic function

$$
\left.(-1)^{\left|w_{(1)}\right|\left|w_{(2)}\right|}\left\langle w_{(0)}^{\prime}, \mathcal{Y}_{3}\left(w_{(2)}, x_{2}\right) \mathcal{Y}_{4}\left(w_{(1)}, x_{1}\right) w_{(3)}\right\rangle\right|_{x_{1}=z_{1}, x_{2}=z_{2}}
$$

of $z_{1}$ and $z_{2}$ in the region $\left|z_{2}\right|>\left|z_{1}\right|>0$ are analytic extensions of each other.

In [H4], the notion of intertwining operator algebra was introduced (see also [H6] and [H7]). This notion has the following generalization: 
Definition 4.3 An $\mathbb{R} \times \mathbb{Z}_{2}$-graded vector space $W=\coprod_{n \in \mathbb{R}} W_{(n)}$, together with a finite set $\mathcal{A}$ with a distinguished element e, a subspace $\mathcal{V}_{a_{1} a_{2}}^{a_{3}}$ of the space of linear maps from $W^{a_{1}} \otimes W^{a_{2}} \rightarrow W^{a_{3}}\{x\}$ for $a_{1}, a_{2}, a_{3} \in \mathcal{A}$, two distinguished vectors, the vacuum 1 and the Virasoro element $\omega$ of $W^{e}$, is called an intertwining operator superalgebra if $W$ together with the other data satisfies all the axioms for intertwining operator algebras except that commutativity (or skew-symmetry) for intertwining operators is replaced by the corresponding commutativity (or skew-symmetry) for intertwining operators for vertex operator superalgebras. Note that there is a unique intertwining operator $Y$ of type $\left(\begin{array}{c}W^{e} \\ W^{e} W^{e}\end{array}\right)$ such that $\left(W^{e}, Y, \mathbf{1}, \omega\right)$ is a vertex operator subalgebra of $W$. If there is an element $\tau \in W_{e}$ such that $(V, Y, \mathbf{1}, \tau)$ is an $N=1$ superconformal vertex operator algebra, then $W$ together with all the other data is called an $N=1$ superconformal intertwining operator superalgebra.

The intertwining operator superalgebra just defined is denoted

$$
\left(W, \mathcal{A},\left\{\mathcal{V}_{a_{1} a_{2}}^{a_{3}}\right\}, \mathbf{1}, \tau\right)
$$

or simply $W$. Theorem 3.5 in [H4 can be modified easily to incorporate the signs and to apply to the cases discussed in the present paper. Applying this modification to $V$, we obtain the following:

Theorem 4.4 Assume in addition that $V$ is rational. Let $\mathcal{A}=\left\{a_{i}\right\}_{i=1}^{m}$ be the set of all equivalence classes of irreducible $V$-modules. Let $W^{a_{1}}, \ldots, W^{a_{m}}$ be representatives of $a_{1}, \ldots, a_{m}$, respectively. Let $W=\coprod_{i=1}^{m} W^{a_{i}}$, and let $\mathcal{V}_{a_{1} a_{2}}^{a_{3}}$, for $a_{1}, a_{2}, a_{3} \in \mathcal{A}$, be the space of intertwining operators of type $\left(\begin{array}{c}W^{a_{3}} \\ W^{a_{1}} W^{a_{2}}\end{array}\right)$. Then $\left(W, \mathcal{A},\left\{\mathcal{V}_{a_{1} a_{2}}^{a_{3}}\right\}, \mathbf{1}, \tau\right)$ (where $\mathbf{1}, \tau$ are the vacuum and Neuve-Schwarz element of $V$, respectively) is an $N=1$ superconformal intertwining operator superalgebra.

In particular, we have:

Theorem 4.5 For any integers $p, q$ larger than 1 such that $p-q \in 2 \mathbb{Z}$ and $(p-q) / 2$ and $q$ relatively prime to each other, the direct sum

$$
\coprod_{m, n \in \mathbb{Z}, m-n \in 2 \mathbb{Z}, 0<m<p, 0<n<q} L_{\mathfrak{n s}}\left(c_{p, q}, h_{p, q}^{m, n}\right)
$$

together with the finite set

$$
\{m, n \in \mathbb{Z}, m-n \in 2 \mathbb{Z}, 0<m<p, 0<n<q\},
$$


the spaces of intertwining operators of type

$$
\left(\begin{array}{c}
L_{\mathfrak{n s}}\left(c_{p, q}, h_{p, q}^{m_{3}, n_{3}}\right) \\
L_{\mathfrak{n} \mathfrak{s}}\left(c_{p, q}, h_{p, q}^{m_{1}, n_{1}}\right) L_{\mathfrak{n} \mathfrak{s}}\left(c_{p, q}, h_{p, q}^{m_{2}, n_{2}}\right)
\end{array}\right)
$$

for $m_{i}, n_{i} \in \mathbb{Z}, m_{i}-n_{i} \in 2 \mathbb{Z}, 0<m_{i}<p, 0<n_{i}<q, i=1,2$, and the vacuum and the Neveu-Schwarz elements of $L_{\mathfrak{n s}}\left(c_{p, q}, 0\right)$ is an $N=1$ superconformal intertwining operator superalgebra.

Now we discuss the vertex tensor category structures. Recall the sphere partial operad $K=\{K(j)\}_{j \in \mathbb{N}}$, the vertex partial operads $\tilde{K}^{c}=\left\{\tilde{K}^{c}(j)\right\}_{j \in \mathbb{N}}$ of central charge $c \in \mathbb{C}$ constructed in $[\mathrm{H} 5$ and the definition of vertex tensor category in HL2 and HL7. For any $c \in \mathbb{C}$ and $j \in \mathbb{N}, \tilde{K}^{c}(j)$ is a trivial holomorphic line bundle over $K(j)$ and we have a canonical holomorphic section $\psi_{j}$. Given a vertex tensor category, we have, among other things, a tensor product bifunctor $\bigotimes_{\tilde{Q}}$ for each $\tilde{Q} \in \tilde{K}^{c}(2)$. In particular, $\psi_{2}(P(z)) \in$ $\tilde{K}^{c}(2)$ and thus there is a tensor product bifunctor $\bigotimes_{\psi_{2}(P(z))}$.

Note that $\bigotimes_{P(z)}$ constructed in [HL5] can be generalized without any difficulty to categories of modules for vertex operator superalgebras. By Proposition 2.7 and Theorem [3.5, and Theorem 3.2 and Corollary 3.3 in [H2], we obtain:

Theorem 4.6 Let $c$ be the central charge of $V$. Then the category of $V$ modules has a natural structure of vertex tensor category of central charge $c$ such that for each $z \in \mathbb{C}^{\times}$, the tensor product bifunctor $\bigotimes_{\psi_{2}(P(z))}$ associated with $\psi_{2}(P(z)) \in \tilde{K}^{c}(2)$ is equal to generalization to the category $V$-modules of $\otimes_{P(z)}$ constructed in HLS.

Combining Theorem 4.6 with Theorem 4.4 in [HL2 (see [HL7 for the proof), we obtain:

Corollary 4.7 The category of $V$-modules has a natural structure of braided tensor category such that the tensor product bifunctor is $\bigotimes_{P(1)}$. In particular, the category of $L_{\mathfrak{n s}}\left(c_{p_{1}, q_{1}}, 0\right) \otimes \cdots \otimes L_{\mathfrak{n} \mathfrak{s}}\left(c_{p_{m}, q_{m}}, 0\right)$-modules has a natural structure of braided tensor category.

In particular, the special case $V=L_{\mathfrak{n} \mathfrak{s}}\left(c_{p, q}, 0\right)$ gives: 
Theorem 4.8 For any integers $p, q$ larger than 1 such that $p-q \in 2 \mathbb{Z}$ and $(p-q) / 2$ and $q$ relatively prime to each other, the category of modules for the $N=1$ Neveu-Schwarz Lie superalgebra equivalent to $L_{\mathfrak{n s}}\left(c_{p, q}, h_{p, q}^{m, n}\right)$, $m, n \in \mathbb{Z}, m-n \in 2 \mathbb{Z}, 0<m<p, 0<n<q$, has a natural structure of braided tensor category such that the tensor product bifunctor is $\bigotimes_{P(1)}$.

\section{Appendix: An example}

In this appendix we give a concrete example. Using this example, we present a way to calculate the fusion rules for minimal models and also show how to derive explicit differential equations for matrix coefficients.

By Theorem 2.1, for integers $p, q$ larger than 1 such that $p-q \in 2 \mathbb{Z}$ and $(p-q) / 2$ and $q$ are relatively prime to each other, all irreducible representations of vertex operator superalgebra $L_{\mathfrak{n} \mathfrak{s}}\left(c_{p, q}, 0\right)$ are given by the set $L_{\mathfrak{n s}}\left(c_{p, q}, h_{p, q}^{m, n}\right)$ where $m, n \in \mathbb{N}, 0<m<p, 0<n<q$ and $m-n \in 2 \mathbb{Z}$. We consider the special case $c=-11 / 14$, that is, $p=7$ and $q=3$. Then vertex operator superalgebra $L_{\mathfrak{n} \mathfrak{s}}(-11 / 14,0)$ has three inequivalent irreducible modules $L_{\mathfrak{n s}}(-11 / 14,2 / 7), L_{\mathfrak{n} \mathfrak{s}}(-11 / 14,-1 / 14)$ and itself. The following fact follows from the representation theory (see As ) of $N=1$ Neveu-Schwarz Lie superalgebra:

Proposition 5.1 1. The irreducible module $L_{\mathfrak{n s}}(-11 / 14,0)$ is equivalent to $V_{\mathfrak{n} \mathfrak{s}}(-11 / 14,0) / J^{1}$, where $J^{1}$ is a submodule generated by a (singular) vector of weight 6 .

2. The irreducible module $L_{\mathfrak{n s}}(-11 / 14,2 / 7)$ is equivalent to

$$
M_{\mathfrak{n} \mathfrak{s}}(-11 / 14,2 / 7) /\left(J^{2}+J^{3}\right)
$$

where $J^{2}$ and $J^{3}$ are submodules generated by (singular) vectors of weights 2 and $5 / 2$, respectively.

3. The irreducible module $L_{\mathfrak{n} \mathfrak{s}}(-11 / 14,-1 / 14)$ is equivalent to

$$
M_{\mathfrak{n s}}(-11,14,-1 / 14) /\left(J^{4}+J^{5}\right)
$$

where $J^{4}$ and $J^{5}$ are submodules generated by (singular) vectors of weights 4 and $3 / 2$, respectively. 
The proof of this result is easy and is omitted.

Now we assume that the reader is familiar with the theory of Zhu's algebra associated to a vertex operator algebra (see [Z]). It has been shown in [KWan] that $A\left(V_{\mathfrak{n s}}(c, 0)\right)$ (Zhu's algebra associated to $\left.V_{\mathfrak{n s}}(c, 0)\right)$ is isomorphic to $\mathbb{C}[x]$ where $x=[\omega]$. Using this information and Conclusion 1 in Proposition 5.1, we see that $A\left(L_{\mathfrak{n s}}(-11 / 14,0)\right)$ is isomorphic to $\mathbb{C}[x] /\langle x(x-2 / 7)(x+1 / 14)\rangle$.

Next we use Frenkel-Zhu's formula in FZ to calculate the fusion rules. First we have the following result which can be obtained by using the results in KWan:

Proposition 5.2 Let $A\left(M_{\mathfrak{n s}}(c, h)\right)$ be the $A\left(V_{\mathfrak{n s}}(c, 0)\right)$-bimodule associated to the $V_{\mathfrak{n s}}(c, 0)$-module $M_{\mathfrak{n} \mathfrak{s}}(c, h)$ with the left and right action given by

$$
\begin{aligned}
& a * m=\operatorname{Res}_{x} Y(a, x) \frac{(1+x)^{\operatorname{deg}(a)}}{x} m, \\
& m * a=\operatorname{Res}_{x} Y(a, x) \frac{(1+x)^{\operatorname{deg}(a)-1}}{x} m,
\end{aligned}
$$

respectively, for $a \in V_{0}, m \in A\left(M_{\mathfrak{n} \mathfrak{s}}(c, h)\right)$ and with trivial action for $a \in V_{1}$, $m \in A\left(M_{\mathfrak{n} \mathfrak{s}}(c, h)\right)$. Then we have:

1. The $A\left(V_{\mathfrak{n s}}(c, 0)\right)$-bimodule $A\left(M_{\mathfrak{n s}}(c, h)\right)$ is equivalent to

$$
\mathbb{C}[x, y] \oplus \mathbb{C}[x, y] v
$$

where $x=[L(0)+2 L(-1)+L(-2)], y=[L(-1)+L(-2)]$ and $v=$ $[G(-1 / 2)]$.

2. We have the following action of $A\left(V_{\mathfrak{n} \mathfrak{s}}(c, 0)\right) \cong \mathbb{C}[y]$ on $A\left(M_{\mathfrak{n} \mathfrak{s}}(c, h)\right) \cong$ $\mathbb{C}[x, y] \oplus \mathbb{C}[x, y] v:$

$$
\begin{array}{r}
y *\left(x^{k} y^{l}\right)=x^{k+1} y^{l}, \quad\left(x^{k} y^{l}\right) * y=x^{k} y^{l+1}, \\
y *\left(x^{k} y^{l} v\right)=x^{k+1} y^{l} v, \quad\left(x^{k} y^{l} v\right) * y=x^{k} y^{l+1} v .
\end{array}
$$

The proof of this result is straightforward and is omitted. From this result, we see that $A\left(M_{\mathfrak{n s}}(c, h)\right)$ has $\mathbf{Z}_{2}$-grading:

$$
A\left(M_{\mathfrak{n s}}(c, h)\right)=A\left(M_{\mathfrak{n} \mathfrak{s}}(c, h)\right)^{+} \oplus A\left(M_{\mathfrak{n} \mathfrak{s}}(c, h)\right)^{-}
$$

where

$$
A\left(M_{\mathfrak{n s}}(c, h)\right)^{+}=\mathbb{C}[x, y]
$$


and

$$
A\left(M_{\mathfrak{n} \mathfrak{s}}(c, h)\right)^{-}=\mathbb{C}[x, y] v .
$$

Using this result, we identify $A\left(L_{\mathfrak{n s}}(-11 / 14,2 / 7)\right)$ as follows: It is not hard to see that $J^{2}$ is generated by the vector

$$
\left(8 L(-2)-21 L(-1)^{2}+21 G(-3 / 2) G(-1 / 2)\right) w
$$

and $J^{3}$ is generated by

$$
\left(L(-2) G(-1 / 2)-\frac{25}{14} G(-5 / 2)-\frac{7}{6} L(-1)^{2} G(-1 / 2)+\frac{3}{2} L(-1) G(-3 / 2)\right) w,
$$

where $w$ is a lowest weight vector in $M_{\mathfrak{n s}}(-11 / 14,2 / 7)$.

Since for any $A\left(L_{\mathfrak{n s}}(-11 / 14,0)\right)$-module $M$ and any submodule $I$ of $M$, $A(M / I)$ is equivalent to $A(M) / A(I)$, we obtain by using Proposition 5.2 and calculations that $A\left(L_{\mathfrak{n s}}(-11 / 14,2 / 7)\right)$ is equivalent to $\mathbb{C}[x, y] / I_{1} \oplus \mathbb{C}[x, y] v / I_{2} v$ where

$$
\begin{aligned}
& I_{1}=\left\langle-21(x-y)^{2}+4(x+y)+\frac{4}{7},(x-y)\left(49(x-y)^{2}-84(x+y)+20\right)\right\rangle, \\
& I_{2}=\left\langle-\frac{7}{6}(x-y)^{2}+\frac{1}{2}(x+y)+\frac{1}{24},-\frac{75}{28}+25(x+y)-21(x-y)^{2}\right\rangle .
\end{aligned}
$$

Note that $\operatorname{dim}_{\mathbb{C}} A\left(L_{\mathfrak{n} \mathfrak{s}}(-11 / 14,2 / 7)\right)=4$.

We now apply Frenkel-Zhu's formula for the fusion rules, that is, the dimensions of the spaces of intertwining operators. It is proved in [FZ] (see also $[\mathbb{L}]$ ) that for suitable vertex operator algebra $V$ and any $V$-modules $W_{1}$, $W_{2}$ and $W_{3}$, the fusion rule

$$
\mathcal{N}_{W_{1} W_{2}}^{W_{3}}=\operatorname{dim} \operatorname{Hom}_{A(V)}\left(A\left(W_{1}\right) \otimes_{A(V)} W_{2}(0), W_{3}(0)\right),
$$

where $W_{i}(0), i=1,2,3$, are the top level of $W_{i}$, respectively, equipped with $A(V)$-module structures.

We have shown in the proof of Proposition 2.2 that any intertwining operator $\mathcal{Y}$ among irreducible modules is uniquely determined by $\mathcal{Y}\left(w_{1}, z\right)$ and $\mathcal{Y}\left(G(-1 / 2) w_{1}, z\right)$ where $w_{(1)}$ is a lowest weight vector. Now we have actually reproved this fact using Frenkel-Zhu's theory. In fact from the $\mathbb{Z}_{2^{-}}$ grading of $A(W)=A(W)^{0} \oplus A(W)^{1}$, it follows that

$$
\begin{aligned}
& \operatorname{Hom}_{A(V)}\left(A\left(W_{1}\right) \otimes_{A(V)} W_{2}(0), W_{3}(0)\right) \cong \\
& \operatorname{Hom}_{A(V)}\left(A\left(W_{1}\right)^{0} \otimes_{A(V)} W_{2}(0), W_{3}(0)\right) \\
& \oplus \operatorname{Hom}_{A(V)}\left(A\left(W_{1}\right)^{-} \otimes_{A(V)} W_{2}(0), W_{3}(0)\right) .
\end{aligned}
$$


Thus one can define in an obvious way $\left(\mathcal{N}_{W_{1} W_{2}}^{W_{3}}\right)^{0}$ and $\left(\mathcal{N}_{W_{1} W_{2}}^{W_{3}}\right)^{1}$. We have proved in Proposition 2.2 that $\mathcal{N}_{W_{1} W_{2}}^{W_{3}} \leq 2$. A natural question is: Is it true that $\left(\mathcal{N}_{W_{1} W_{2}}^{W_{3}}\right)^{0} \leq 1$ and $\left(\mathcal{N}_{W_{1} W_{2}}^{W_{3}}\right)^{1} \leq 1$ ?

Let us go back to our example. For convenience, we shall denote the fusion rules among irreducible modules $L_{\mathfrak{n s}}\left(-11 / 14, h_{1}\right), L_{\mathfrak{n s}}\left(-11 / 14, h_{2}\right)$ and $L_{\mathfrak{n s}}\left(-11 / 14, h_{3}\right)$ for $L_{\mathfrak{n s}}(-11 / 14,0)$ by $\mathcal{N}_{h_{1} h_{2}}^{h_{3}}$. By using the formula above and this notation, we obtain

$$
\begin{aligned}
\mathcal{N}_{2 / 7,2 / 7}^{0} & =1, \\
\mathcal{N}_{2 / 7,2 / 7}^{-1 / 14} & =1, \\
\mathcal{N}_{2 / 7}^{2 / 7}= & =0, \\
\mathcal{N}_{2 / 7,-1 / 14}^{0} & =0, \\
\mathcal{N}_{2 / 7}^{2 / 7}-1 / 14 & =1, \\
\mathcal{N}_{2 / 7,-1 / 14}^{2 / 7} & =1, \\
\mathcal{N}_{2 / 7,-1 / 14}^{-1 / 14} & =1, \\
\mathcal{N}_{2 / 7,-1 / 14}^{-1 / 14} & =1,
\end{aligned}
$$

in addition to the obvious fusion rules. In the proceeding section, we have proved that there is a braided tensor category structure on the category of modules for $L_{\mathfrak{n s}}\left(c_{p, q}, 0\right)$. Using the tensor product notation $\otimes=\nabla_{P(1)}$ and using $\cong$ as an abbreviation of the phrase "is isomorphic to," we have the following decompositions of the tensor product modules:

$$
\begin{aligned}
& L_{\mathfrak{n s}}(-11 / 14,2 / 7) \otimes L_{\mathfrak{n s}}(-11 / 14,2 / 7) \\
& \cong L_{\mathfrak{n s}}(-11 / 14,-1 / 14) \oplus L_{\mathfrak{n s}}(-11 / 14,0), \\
& L_{\mathfrak{n} \mathfrak{s}}(-11 / 14,2 / 7) \otimes L_{\mathfrak{n \mathfrak { s }}}(-11 / 14,-1 / 14) \\
& \cong L_{\mathfrak{n s}}(-11 / 14,2 / 7) \oplus L_{\mathfrak{n s}}(-11 / 14,-1 / 14), \\
& L_{\mathfrak{n s}}(-11 / 14,-1 / 14) \otimes L_{\mathfrak{n s}}(-11 / 14,-1 / 14) \\
& \cong L_{\mathfrak{n s}}(-11 / 14,0) \oplus L_{\mathfrak{n s}}(-11 / 14,-1 / 14) \oplus L_{\mathfrak{n s}}(-11 / 14,2 / 7) .
\end{aligned}
$$

Finally we discuss the differential equations. Let

$$
\mathcal{Y}_{1}, \mathcal{Y}_{2} \in \mathcal{V}_{L_{\mathfrak{n} \mathfrak{s}}(-11 / 14,-1 / 14)}^{L_{\mathfrak{n}}(-11 / 14,2 / 7)} L_{\mathfrak{n} \mathfrak{s}}(-11 / 14,2 / 7) .
$$

Our goal is to derive differential equations from which we can find the sums of the series

$$
\left\langle w_{(4)}^{\prime}, \mathcal{Y}_{1}\left(w_{(1)}, z_{1}\right) \mathcal{Y}_{2}\left(w_{(2)}, z_{2}\right) w_{(3)}\right\rangle,
$$


where $w_{(1)}, w_{(2)} \in L_{\mathfrak{n s}}(-11 / 14,-1 / 14), w_{(3)} \in L_{\mathfrak{n s}}(-11 / 14,2 / 7)$ and $w_{(4)}^{\prime} \in$ $L^{\prime}(-11 / 14,2 / 7)$ are the lowest weight vectors in these modules. We will see that even with singular vectors of weight 2 , we have highly non-trivial differential equations.

Since

$$
\begin{aligned}
& \left\langle w_{(4)}^{\prime}, \mathcal{Y}_{1}\left(w_{(1)}, z_{1}\right) \mathcal{Y}_{2}\left(w_{(2)}, z_{2}\right)\left(8 L(-2)-21 L(-1)^{2}\right.\right. \\
& \left.+21 G(-3 / 2) G(-1 / 2)) w_{(3)}\right\rangle=0, \\
& \left\langle w_{(4)}^{\prime}, \mathcal{Y}_{1}\left(G(-1 / 2) w_{(1)}, z_{1}\right) \mathcal{Y}_{2}\left(G(-1 / 2) w_{(2)}, z_{2}\right)\left(8 L(-2)-21 L(-1)^{2}\right.\right. \\
& \left.+21 G(-3 / 2) G(-1 / 2)) w_{(3)}\right\rangle=0,
\end{aligned}
$$

we obtain

$$
\begin{gathered}
\left(-8\left(\frac{1}{14 z_{1}^{2}}+\frac{1}{14 z_{2}^{2}}\right)-21\left(\partial_{z_{1}}+\partial_{z_{2}}\right)^{2}-29\left(\frac{\partial_{z_{1}}}{z_{1}}+\frac{\partial_{z_{2}}}{z_{2}}\right)\right) Q_{0,0}\left(z_{1}, z_{2}\right) \\
+21\left(\frac{1}{z_{1}}-\frac{1}{z_{2}}\right) Q_{1,1}\left(z_{2}, z_{2}\right)=0 \\
\left(\frac{3}{7 z_{1}^{2}}+\frac{3}{7 z_{2}^{2}}-21\left(\partial_{z_{1}}+\partial_{z_{2}}\right)^{2}-29\left(\frac{\partial_{z_{1}}}{z_{1}}+\frac{\partial_{z_{2}}}{z_{2}}\right)\right) Q_{1,1}\left(z_{1}, z_{2}\right) \\
+\left(\left(\frac{1}{z_{2}}-\frac{1}{z_{1}}\right) \partial_{z_{1}} \partial_{z_{2}}+\frac{1}{7}\left(\frac{\partial_{z_{1}}}{z_{2}^{2}}-\frac{\partial_{z_{2}}}{z_{1}^{2}}\right)\right) Q_{0,0}\left(z_{1}, z_{2}\right)=0
\end{gathered}
$$

Note that this is a system of regular singular points.

Since

$$
\begin{aligned}
& Q_{0,0}\left(z_{1}, z_{2}\right)=z_{1}^{1 / 14} z_{2}^{1 / 14} R_{0,0}\left(\left(z_{2} / z_{1}\right)^{1 / 2}\right), \\
& Q_{1,1}\left(z_{1}, z_{2}\right)=z_{1}^{1 / 14-1 / 2} z_{2}^{1 / 14-1 / 2} R_{1,1}\left(\left(z_{2} / z_{1}\right)^{1 / 2}\right),
\end{aligned}
$$

we obtain the following system for $R_{0,0}(z)$ and $R_{1,1}(z)$ :

$$
\begin{aligned}
& R_{0,0}^{\prime \prime}(z)-\frac{-43 z^{-1}+100 z-54 z^{3}}{21\left(1-z^{2}\right)^{2}} R_{0,0}^{\prime}(z) \\
& \quad-\frac{23 z^{2}+23 z^{-2}-6}{147\left(1-z^{2}\right)^{2}} R_{0,0}(z)+\frac{4}{z\left(1-z^{2}\right)} R_{1,1}(z)=0 \\
& R_{1,1}^{\prime \prime}(z)-\frac{100 z-117 z^{3}-z^{-1}}{21\left(1-z^{2}\right)^{2}} R_{1,1}^{\prime}(z) \\
& \quad-\frac{324}{21\left(1-z^{2}\right)^{2}} R_{1,1}(z)+\frac{83 z-203 z^{3}+114 z^{5}-21+42 z^{2}-21 z^{4}}{21 z\left(z^{2}-1\right)^{3}} R_{0,0}^{\prime}(z) \\
& \quad-\frac{37 z^{4}+6 z^{2}+37}{147 z\left(z^{2}-1\right)^{3}} R_{0,0}(z)=0 .
\end{aligned}
$$


Note that this system is analytic in the region $0<|z|<1$ and has a regular singularity at $z=0$. Using the standard method, one can prove the convergence of power series solutions of (5.3)-(5.4).

Similarly, from

$$
\begin{aligned}
\left\langle w_{(4)}^{\prime}, \mathcal{Y}_{1}\left(G(-1 / 2) w_{(1)}, z_{1}\right) \mathcal{Y}_{2}\left(w_{(2)}, z_{2}\right)\left(8 L(-2)-21 L(-1)^{2}\right.\right. & \left.+21 G(-3 / 2) G(-1 / 2)) w_{(3)}\right\rangle=0 \\
& +21 L(-1)^{2} \\
\left\langle w_{(4)}^{\prime}, \mathcal{Y}_{1}\left(w_{(1)}, z_{1}\right) \mathcal{Y}_{2}\left(G(-1 / 2) w_{(2)}, z_{2}\right)(8 L(-2)-21 L(-3) G(-1 / 2)) w_{(3)}\right\rangle=0 & \\
+ & 21 G(-3 / 2) G
\end{aligned}
$$

we obtain the following system:

$$
\begin{aligned}
&\left(\left(\frac{3}{7 z_{1}^{2}}-\frac{4}{7 z_{2}^{2}}\right)-21\left(\partial_{z_{1}}\right.\right.\left.\left.+\partial_{z_{2}}\right)^{2}-29\left(\frac{\partial_{z_{1}}}{z_{1}}+\frac{\partial_{z_{2}}}{z_{2}}\right)\right) Q_{1,0}\left(z_{1}, z_{2}\right) \\
&+\left(21\left(\frac{\partial_{z_{1}}}{z_{2}}-\frac{\partial_{z_{1}}}{z_{1}}\right)-\frac{21}{7 z_{1}^{2}}\right) Q_{0,1}\left(z_{2}, z_{2}\right)=0 \\
&\left(\left(\frac{3}{7 z_{2}^{2}}-\frac{4}{7 z_{1}^{2}}\right)-21\left(\partial_{z_{1}}+\partial_{z_{2}}\right)^{2}-29\left(\frac{\partial_{z_{1}}}{z_{1}}+\frac{\partial_{z_{2}}}{z_{2}}\right)\right) Q_{0,1}\left(z_{1}, z_{2}\right) \\
&+\left(21\left(\frac{\partial_{z_{2}}}{z_{1}}-\frac{\partial_{z_{2}}}{z_{2}}\right)-\frac{21}{7 z_{2}^{2}}\right) Q_{1,0}\left(z_{2}, z_{2}\right)=0 .
\end{aligned}
$$

Since

$$
\begin{aligned}
& Q_{1,0}\left(z_{1}, z_{2}\right)=z_{1}^{1 / 14-1 / 2} z_{2}^{1 / 14} R_{1,0}\left(\left(z_{2} / z_{1}\right)^{1 / 2}\right), \\
& Q_{0,1}\left(z_{1}, z_{2}\right)=z_{1}^{1 / 14} z_{2}^{1 / 14-1 / 2} R_{0,1}\left(\left(z_{2} / z_{1}\right)^{1 / 2}\right),
\end{aligned}
$$

we obtain a system for $R_{1,0}(z)$ and $R_{0,1}(z)$ :

$$
\begin{aligned}
& R_{1,0}^{\prime \prime}(z)-\frac{168 z-118 z^{3}-116 z^{-1}}{42\left(z^{2}-1\right)^{2}} R_{1,0}(z) \\
& \quad-\frac{\frac{36}{147}-\frac{348}{147} z^{-2}+\frac{14700}{28182}}{\left(z^{2}-1\right)^{2}} R_{1,0}(z)-\frac{2}{z^{2}-1} R_{0,1}^{\prime}(z)- \\
& \quad \frac{2 z^{-1}-6 z}{7\left(z^{2}-1\right)^{2}} R_{0,1}(z)=0 \\
& R_{0,1}^{\prime \prime}(z)+\frac{-425 z^{-1}+424 z^{3}}{7\left(z^{2}-1\right)^{2}} R_{0,1}(z) \\
& \quad-\frac{12 z^{-2}-16 z^{2}}{147\left(z^{2}-1\right)^{2}} R_{0,1}(z)-\frac{2}{z^{2}-1} R_{1,0}(z)+
\end{aligned}
$$




$$
\frac{-84 z+96 z^{-1}}{21\left(z^{2}-1\right)^{2}} R_{1,0}(z)=0 .
$$

\section{References}

[Ad] D. Adamović, Rationality of Neveu-Schwarz vertex operator superalgebras, Internat. Math. Res. Notices 1997 (1997), 865-874.

[AM] D. Adamović and A. Milas, Vertex operator algebras associated to modular invariant representations for $A_{1}^{(1)}$, Math. Res. Lett. 2 (1995), 563-575.

[As] A. Astashkevich, On the structure of Verma modules over Virasoro and Neveu-Schwarz algebras, Comm. Math. Phys. 186 (1997), 531562.

[Ba1] K. Barron, A supergeometric interpretation of vertex operator superalgebras, Internat. Math. Res. Notices 1996 (1996), 409-430.

[Ba2] K. Barron, The supergeometric interpretation of vertex operator superalgebras, Ph.D. thesis, Rutgers University, 1996.

[Bo] R. E. Borcherds, Vertex algebras, Kac-Moody algebras, and the Monster, Proc. Natl. Acad. Sci. USA 83 (1986), 3068-3071.

[C1] J. L. Cardy, Effect of boundary conditions on the operator content of two-dimensional conformally invariant theories, Nucl. Phys. B275 (1986), 200-218.

[C2] J. L. Cardy, Boundary conditions, fusion rules and the Verlinde formula, Nucl. Phys. B324 (1989), 581-596.

[D] L. Dixon, Some world-sheet properties of superstring compactifications, on orbifolds and otherwise, in: Superstrings, Unified Theories and Cosmology 1987 (Trieste, 1987), ICTP Ser. Theoret. Phys., Vol. 4, World Sci. Publishing, Teaneck, NJ, 1988, 67-126.

[DGH] L. Dixon, P. Ginsparg and J. Harvey, Beauty and the beast: superconformal symmetry in a Monster module, Comm. Math. Phys. 119 (1988), 221-241 
[DGM] L. Dolan, P. Goddard and P. Montague, Conformal field theory of twisted vertex operators, Nucl. Phys. B338 (1990), 529-601.

[DMZ] C. Dong, G. Mason, Y. Zhu, Discrete series of the Virasoro algebra and the moonshine module, in: Algebraic groups and their generalizations: quantum and infinite-dimensional methods (University Park, PA, 1991), Proc. Sympos. Pure Math., Vol. 56, Part 2, Amer. Math. Soc., Providence, RI, 1994, 295-316.

[FHL] I. B. Frenkel, Y.-Z. Huang and J. Lepowsky, On axiomatic approaches to vertex operator algebras and modules, preprint, 1989; Memoirs Amer. Math. Soc. 104, 1993.

[FLM1] I. B. Frenkel, J. Lepowsky and A. Meurman, A natural representation of the Fischer-Griess monster with the modular function $J$ as character, Proc. Natl. Acad. Sci. USA 81 (1984), 3256-3260.

[FLM2] I. B. Frenkel, J. Lepowsky, and A. Meurman, Vertex operator algebras and the Monster, Pure and Appl. Math., 134, Academic Press, New York, 1988.

[FZ] I. B. Frenkel and Y. Zhu, Vertex operator algebras associated to representations of affine and Virasoro algebras, Duke Math. J. 66 (1992), 123-168.

[FS] J. Fuchs and C. Schweigert, D-brane conformal field theory, hep-th/9801190, to appear.

[Ge1] D. Gepner, Exactly solvable string compactifications on manifolds of rm SU(N) holonomy, Phys. Lett. B199 (1987), 380-388.

[Ge2] D. Gepner, Space-time supersymmetry in compactified string theory and superconformal models, Nucl. Phys. B296 (1988), 757778 .

[GKO] P. Goddard, A. Kent and D. Olive, Unitary representations of the Virasoro and super-Virasoro algebras, Comm. Math. Phys. 103 (1986), 105-119. 
[Gr] B. R. Greene, Aspects of quantum geometry, in: Mirror Symmetry III, Proceedings of the Conference on Complex Geometry and Mirror Symmetry, Montréal, 1995, Stud. Adv. Math., Vol. 10, Amer. Math. Soc., Internat. Press and Centre de Recherches Math., 1-67.

[GP] B. R. Greene and M. R. Plesser, Duality in Calabi-Yau moduli space, Nucl. Phys. B338 (1990), 15-37.

[H1] Y.-Z. Huang, A theory of tensor products for module categories for a vertex operator algebra, IV, J. Pure Appl. Alg., 100 (1995), 173-216.

[H2] Y.-Z. Huang, Virasoro vertex operator algebras, (nonmeromorphic) operator product expansion and the tensor product theory, J. Alg. 182 (1996), 201-234.

[H3] Y.-Z. Huang, A nonmeromorphic extension of the Moonshine module vertex operator algebra, in: Moonshine, the Monster, and related topics (South Hadley, MA, 1994), Contemp. Math., 193, Amer. Math. Soc., Providence, RI, 1996, 123-148.

[H4] Y.-Z. Huang, Intertwining operator algebras, genus-zero modular functors and genus-zero conformal field theories, in: Operads: Proceedings of Renaissance Conferences, ed. J.-L. Loday, J. Stasheff, and A. A. Voronov, Contemporary Math., 202, Amer. Math. Soc., Providence, 1997, 335-355.

[H5] Y.-Z. Huang, Two-dimensional conformal geometry and vertex operator algebras, Progress in Mathematics, Vol. 148, Birkhäuser, Boston, 1997.

[H6] Y.-Z. Huang, Genus-zero modular functors and intertwining operator algebras, Internat. J. Math. 9 (1998), 845-863.

[H7] Y.-Z. Huang, Generalized rationality and a generalized Jacobi identity for intertwining operator algebras, to appear.

[HL1] Y.-Z. Huang and J. Lepowsky, Toward a theory of tensor products for representations of a vertex operator algebra, in: Proc. 20th International Conference on Differential Geometric Methods 
in Theoretical Physics, New York, 1991, ed. S. Catto and A. Rocha, World Scientific, Singapore, 1992, Vol. 1, 344-354.

[HL2] Y.-Z. Huang and J. Lepowsky, Tensor products of modules for a vertex operator algebra and vertex tensor categories, in: Lie Theory and Geometry, in honor of Bertram Kostant, ed. R. Brylinski, J.-L. Brylinski, V. Guillemin, V. Kac, Birkhäuser, Boston, 1994, 349383.

[HL3] Y.-Z. Huang and J. Lepowsky, A theory of tensor products for module categories for a vertex operator algebra, I, Selecta Mathematica, New Series 1 (1995), 699-756.

[HL4] Y.-Z. Huang and J. Lepowsky, A theory of tensor products for module categories for a vertex operator algebra, II, Selecta Mathematica, New Series 1 (1995), 757-786.

[HL5] Y.-Z. Huang and J. Lepowsky, A theory of tensor products for module categories for a vertex operator algebra, III, J. Pure Appl. Alg. 100 (1995), 141-171.

[HL6] Y.-Z. Huang and J. Lepowsky, Intertwining operator algebras and vertex tensor categories for affine Lie algebras, Duke Math. J., to appear.

[HL7] Y.-Z. Huang and J. Lepowsky, A theory of tensor products for module categories for a vertex operator algebra, V, to appear.

[KWan] V. Kac and W. Wang, Vertex operator superalgebras and their representations, in: Mathematical aspects of conformal and topological field theories and quantum groups (South Hadley, MA, 1992), Contemp. Math., Vol. 175, 161-191.

[KWak1] V. Kac and M. Wakimoto, Unitarizable highest weight representations of the Virasoro, Neveu-Schwarz and Ramond algebras, in: Conformal groups and related symmetries: physical results and mathematical background (Clausthal-Zellerfield, 1985), Lecture Notes in Phys. Vol. 261, Springer, Berlin, 1986, 345-371. 
[KWak2] V. Kac and M. Wakimoto, Modular invariant representations of infinite-dimensional Lie algebras and superalgebras, Proc. Nat. Acad. Sci. U.S.A. 85 (1988), 4956-4960.

[LVW] W. Lerche, C. Vafa and N. P. Warner, Chiral rings in $N=2$ superconformal theories, Nucl. Phys. B324 (1989), 427-474.

[L] H. Li, Representation theory and tensor product theory for vertex operator algebras, PhD thesis, Rutgers University 1994.

[RS] A. Recknagel and V. Schomerus, D-branes in Gepner models, Nucl. Phys. B531 (1998), 185-225.

[S1] G. B. Segal, The definition of conformal field theory, preprint, 1988.

[S2] G. B. Segal, Two-dimensional conformal field theories and modular functors, in: Proceedings of the IXth International Congress on Mathematical Physics, Swansea, 1988, Hilger, Bristol, 1989, 2237.

[Z] Y. Zhu, Modular invariance of characters of vertex operator algebras, J. Amer. Math. Soc. 9 (1996), 237-302.

Institut des Hautes Études Scientifiques, Le Bois-Marie, 35, Route De Chartres, F-91440 Bures-sur-Yvette, France

and

Department of Mathematics, Rutgers University, 110 Frelinghuysen Rd., Piscataway, NJ 08854-8019 (Permanent address)

E-mail address: yzhuang@math.rutgers.edu

Department of Mathematics, Rutgers University, 110 Frelinghuysen RD., Piscataway, NJ 08854-8019

E-mail address: amilas@math.rutgers.edu 